\title{
1 Evolution in salmon life-history induced 2 by direct and indirect effects of fishing
}

3

4

Authors: Czorlich, Y. ${ }^{1,2,3,4}$, Aykanat T. ${ }^{3}$,Erkinaro, J. ${ }^{2}$, Orell P. ${ }^{2}$, \& Primmer, CR..$^{3,5^{*}}$

\section{Affiliations:}

${ }^{1}$ University of Turku, Department of Biology, FI-20014, Turku, Finland

${ }^{2}$ Natural Resources Institute Finland (Luke), POB 413, FI-90014 Oulu, Finland

${ }^{3}$ University of Helsinki, Organismal \& Evolutionary Biology Research Programme, POB 56, FI00014 Helsinki, Finland

${ }^{4}$ Norwegian Institute for Nature Research (NINA), NO-7485 Trondheim, Norway

${ }^{5}$ University of Helsinki, Institute of Biotechnology, POB 56, FI-00014 Helsinki, Finland

\section{Abstract}

Understanding the drivers of evolution is a fundamental aim in biology. However, identifying the evolutionary impacts of human activities, both direct and indirect, is challenging because of lack of temporal data and limited knowledge of the genetic basis of most traits ${ }^{1}$. Atlantic salmon is a species exposed to intense anthropogenic pressures during its anadromous life cycle ${ }^{2}$. Previous research has shown that salmon age at maturity has evolved towards earlier maturation over the last 40 years, with an $18 \%$ decrease $^{3}$ in the allele associated with late maturation at the large-effect $v g l l 3$ locus ${ }^{4}$; but the drivers of this change remain unknown. Here, we link genetic and phenotypic changes in a large Atlantic salmon population with salmon prey species biomass in the Barents Sea, temperature, and fishing effort in order to identify drivers of age at maturity evolution. We show that age at maturity evolution is associated with two different types of fisheries induced evolution acting in opposing directions: an indirect effect linked with commercial harvest of a salmon prey species (capelin) at sea (selection against late maturation), and a direct effect due to temporal changes in net fishing pressure in the river (surprisingly, selection against early maturation). Although the potential for direct and indirect evolutionary effects of fishing have been acknowledged, empirical evidence for induced changes at the genetic level has been lacking 5 . As capelin are primarily harvested to produce fish meal and oil for aquaculture ${ }^{6}$, we hereby identify an indirect path by which Atlantic salmon aquaculture may negatively affect wild populations. 


\section{Main text}

Identifying biotic and abiotic factors linked with phenotypic change is common but cases demonstrating evolutionary (i.e. genetic) effects remain rare ${ }^{1,7}$. Changes due to indirect effects, whereby the impact of one species on another is mediated by a third species, are prevalent in nature and can have important ecological and evolutionary consequences ${ }^{8}$. Indirect effects are expected to be of more central importance in current-day ecosystem dynamics due to the growing anthropogenic pressure and climate change rate which can strongly influence species, and interactions between them, in complex ways ${ }^{\text {e.g. }}{ }^{9}$. However, detecting indirect evolutionary impacts is extremely challenging in natural ecosystems and has often been neglected ${ }^{8}$.

An example of human activity that can have profound effects, both directly on the target species, and indirectly on entire ecosystems, is fish harvest ${ }^{10}$. Fishing is generally performed at high intensity, over prolonged periods of time, and can be trait selective. It can consequently induce evolution of traits such as size and age at maturity in the target species ${ }^{5,11}$ but also induce larger, ecosystem level, changes, e.g. by reducing the abundance of predators, prey and/or competitors ${ }^{12}$. To date, most research has focused on the effects of harvest on target species at the phenotypic level and cases demonstrating direct effects at the genetic level are rare, as are empirical examples of indirect evolutionary impacts ${ }^{5,13}$. Knowledge of indirect ecological and evolutionary effects is critical for properly evaluating the consequences of different fisheries management strategies ${ }^{10}$.

Atlantic salmon (Salmo salar) have a complex life-history, utilizing both freshwater and marine habitats and thus affect, and are affected by, multiple ecosystems ${ }^{2}$. Sea-age at maturity in Atlantic salmon (the number of years an individual spends in the marine environment before returning to fresh water to spawn), or sea age, is an important life history trait with an evolutionary trade-off between survival and reproduction. Later maturing individuals are larger and have higher reproductive success ${ }^{14}$ but run higher risk of mortality before spawning. Age at maturity has been associated with a major effect locus located in the genome region including the vgll3 gene that explains $40 \%$ of the variation in the trait ${ }^{4}$. We recently demonstrated adaptive evolution towards younger age at maturity at the vgll3 locus over 40 years in a large salmon population in northern Europe $^{3}$. Both sexes experienced a decline in the $v g l l 3$ allele linked with later maturation and only males showed a clear evolutionary response at the phenotypic level, due to vgll3 sex-specific allelic and dominance effects ${ }^{3,4}$. However, the environmental drivers of the evolution in age at maturity remained unknown. 
62

63

64

65

66

67

68

69

70

Here we identify environmental drivers of phenotypic and adaptive changes in age at maturity in the same Atlantic salmon population from the Teno river, in far north Finland and Norway ${ }^{3}$. We investigated ecological and environmental variables potentially affecting the relative fitness (a combination of marine survival and reproductive success) of salmon with different maturation ages, and therefore sizes: fishing effort at sea, fishing effort in the river, sea temperature, and abundance of three key prey species for salmon during their marine migration in the Barents sea ecosystem (capelin Mallotus villosus, herring Clupea harengus and krill, Extended Data Fig. 1). We used (quasi)binomial generalized linear models (GLM) to identify environmental variables linked with temporal variation in vgll3 allele frequencies, and thereby age at maturity, in a 40 year time series (1975 - 2014) consisting of 1319 individuals assigned to the Tenojoki population in ${ }^{3}$ (see methods, Supplementary notes). These analyses indicated that annual riverine net fishing license number (used here as a proxy for annual fishing pressure, see Methods) had the strongest effect on vgll3 allele frequency (Fig. 1). Surprisingly, annual riverine fishing pressure was positively associated with the $v g l l 3 * L$ allele frequency (standardized regression coefficient $\widehat{\beta^{*}}=0.42, \mathrm{~F}_{(1)}=27.79$ and $\mathrm{P}$ value $<0.001$ ) indicating that higher net fishing pressure in the river resulted in higher frequencies of the allele associated with later maturation in salmon and therefore larger size (Extended Data Table 1). We further verified the results using a residual regression approach (de-trending) to account for potentially confounding factors creating temporal trends in the dependent and independent variables, and thus avoid possible spurious associations ${ }^{15}$. The association between annual fishing license number and vgll3 remained in the de-trended model including a significant, negative, year effect ${ }^{\text {e.g. } 3}$, indicating that fishing pressure is also linked with annual vgll3 allele frequency changes around the trend $\left(\mathrm{F}_{(1)}=14.15\right.$ and P-value $<0.001$, Fig. 1, Extended Data Table 1). Capelin biomass in the Barents Sea was also positively associated with the frequency of the vgll3 allele associated with later maturation and larger size $\left(v g l l 3^{*} L\right)$ in salmon in both the normal $\left(\widehat{\beta^{*}}=0.23, \mathrm{~F}_{(1)}=20.77\right.$ and P-value $\left.<0.001\right)$ and de-trended models $\left(\mathrm{F}_{(1)}=10.64\right.$ and P-value $=$ 0.001, Extended Data Table 1). Herring and krill biomass also had a significant effect on vgll3 allele frequencies in a similar direction to capelin (Fig. 1, Extended Data Table 1), however, they did not remain significant in the de-trended model. There was little evidence for associations between the other variables and vgll3 in the GLM (Extended Data Table 1). 


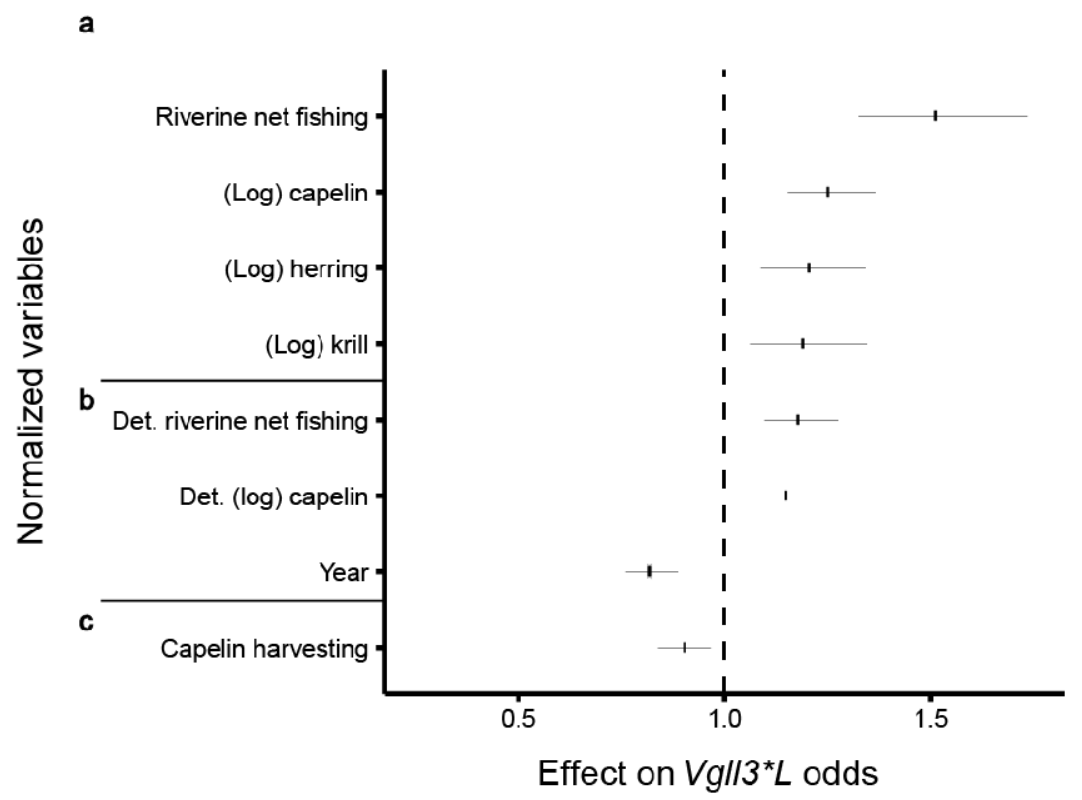

\section{Figure 1: Standardized regression coefficients for variables significantly} associated with $v g l l 3 * L$ odds (later, larger maturation). The estimates come from the $a$, initial quasi-binomial model. $\boldsymbol{b}$, de-trended model. $\boldsymbol{c}$, Monte Carlo Method for assessing mediation (i.e. indirect effects). The dotted line indicates no effect on $v g l l 3 * L$ odds. The error bars correspond to $95 \%$ confidence intervals.

Analyses at the phenotypic level also supported the influence of prey biomass and fishing on Tenojoki Atlantic salmon fitness. After controlling for the vgll3 sex-specific genetic effects, a multinomial model indicated that the probability of observing older, later maturing Atlantic salmon in the river increased with capelin, herring and krill biomass (Supplementary notes, Extended Data Table 3). This result is expected if the abundance of these prey species is positively associated with salmon survival at sea, as only survivors returning back to the river are sampled. However, it can also reflect plastic changes of maturation probabilities. This model also showed that a higher number of riverine net fishing licenses was associated with a higher probability to observe late maturing salmon at the end of the fishing season (Extended Data Table 3), which is expected if net

102 fishing targeted preferentially small, early maturing salmon carrying $v g l l 3^{*} E$. Given that the sea-age 103 at maturity of Tenojoki females is, on average, considerably older than males, and therefore the 104 time spent in the marine environment longer ( 2.8 vs 1.5 years), environmental conditions strongly 105 affecting marine survival are also expected to influence the sex-ratio of adults returning from their 106 migration to spawn. In accordance with this prediction, a binomial model showed that the 107 proportion of returning females increased with prey biomass and riverine net fishing 108 (Supplementary notes; Extended Data Fig. 2, Extended Data Table 3). The effects of net fishing on 
109

110

111 Forage fishes like capelin have important roles in marine ecosystems by enabling energy transfer

112 between lower (plankton) and upper (predators such as large fish, seabirds and mammals) trophic

113 levels ${ }^{6}$. In the Barents Sea, the capelin stock experienced several dramatic collapses during the 40-

114 year study period (Extended Data Fig. 3) due to overexploitation in commercial fisheries combined

115 with predation by herring and $\operatorname{cod}^{16}$. We therefore quantified the potential indirect effects of 116 capelin harvesting (while accounting for other ecosystem interactions) on vgll3 allele frequency 117 dynamics using a multispecies Gompertz model developed in ${ }^{17}$ (Extended Data Fig. 1, Extended

118 Data Fig. 3, Extended Data Fig. 4). The analysis indicated a significant indirect effect of capelin 119 harvest rate on age at maturity evolution in Tenojoki salmon, with a $30 \%$ decrease in the $v g l l{ }^{*} L$ 120 allele odds per harvest rate unit (Monte Carlo method for assessing mediation: $\mathrm{CI}_{95 \%}=[0.116$, 121 0.471]). The evolutionary effect of capelin harvest was the strongest during the early years of the 122 time series, likely because of repeated capelin fisheries closures and fishing effort reduction later 123 on, following the capelin stock collapses (Fig. 2; Extended Data Fig. 5a). This is the same time period when most of the evolutionary changes in male age at maturity occurred ${ }^{3}$.

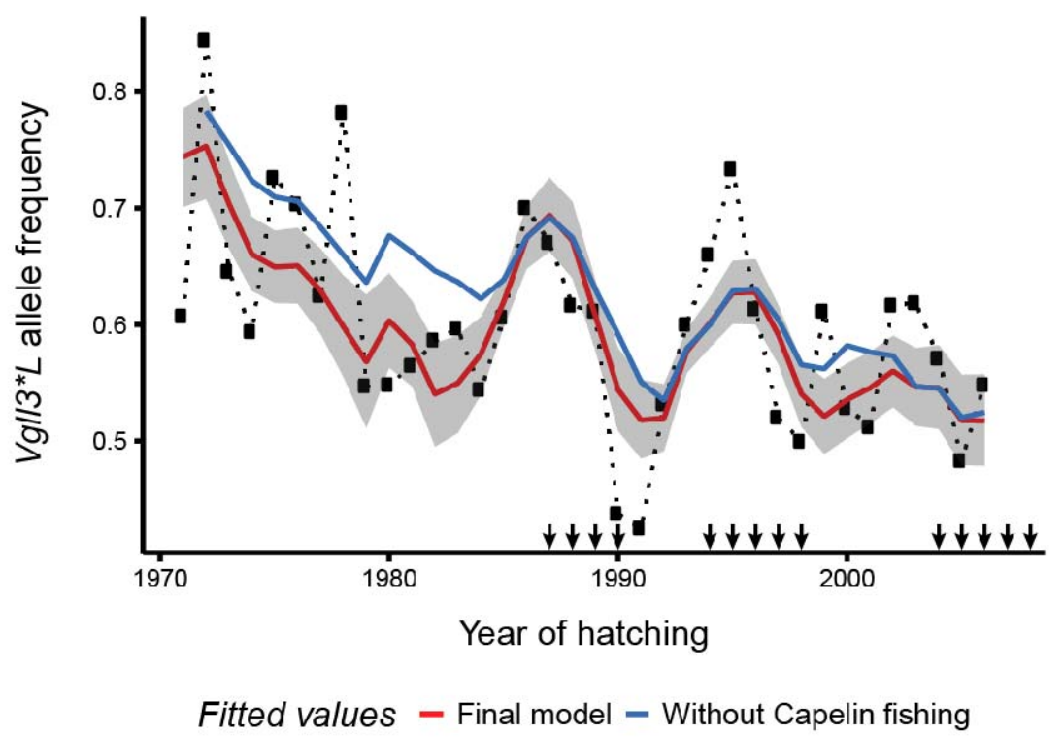

125

126 Figure 2 : Temporal changes in $v g l l 3 * \boldsymbol{L}$ (later maturation) allele frequency. The

127 black dotted line represents the observed data, the red line the allele frequency averaged from model 128

individual fitted values and the blue line the expected $v g l l 3 * L$ allele frequency assuming no capelin fishing 
129 (annual effect on returning salmon). Black arrows indicate years without capelin harvesting (harvest rate <

1300.5 percent). Shaded areas indicate $95 \%$ bootstrap intervals based on 3000 replicates (see methods for 131 details).

132 The direction of the selection pressure in the riverine net fishery may seem counter-intuitive as generally, net fishing is thought to select against large individuals e.g. 18,19. However, net fishing in

134 Tenojoki includes the use of multiple net types with different selectivity: weir, gillnet and driftnet ${ }^{20}$.

135 Sonar data enabled an assessment of the selectivity of each net type (see Methods), and indicated 136 that weir fishing selects against the early maturation allele ( $v g l l 3^{*} E$ ) by capturing proportionally 137 more smaller, and earlier maturing, individuals whereas driftnet and gillnet select against the $138 v g l l 3 * L$ allele (Extended Data Fig. 6, Extended Data Fig. 7). The positive effect of riverine net 139 license number on the $v g l l 3 * L$ allele frequency may thus be explained by the predominant use of 140 weirs (representing 54\% of net catches on average). Over time, the net fishing selective pressure 141 against the $E$ allele is expected to have decreased through a dramatic reduction in the net fishing 142 effort during the time series due to stricter fishing regulations (e.g. net fishing licences and number 143 of weirs were $>30 \%$ lower in 2014 vs. 1976, Extended Data Fig. 5b) and in selectivity due to 144 changes in the relative use of the fishing gears, size distribution and sex-ratio (Fig. 3, 145 Supplementary notes). Overall, the extra mortality at sea of late maturing $v g l l 3 * L$ individuals, 146 which varied over time according to prey biomass, was not sufficiently compensated by their 147 (decreasing) size-induced survival advantage due to riverine net fishing, nor their reproductive 148 advantage due to larger size ${ }^{14}$, thus resulting in the observed overall decrease in the $v g l l{ }^{*} L$ allele 149 frequency (Fig. 2).

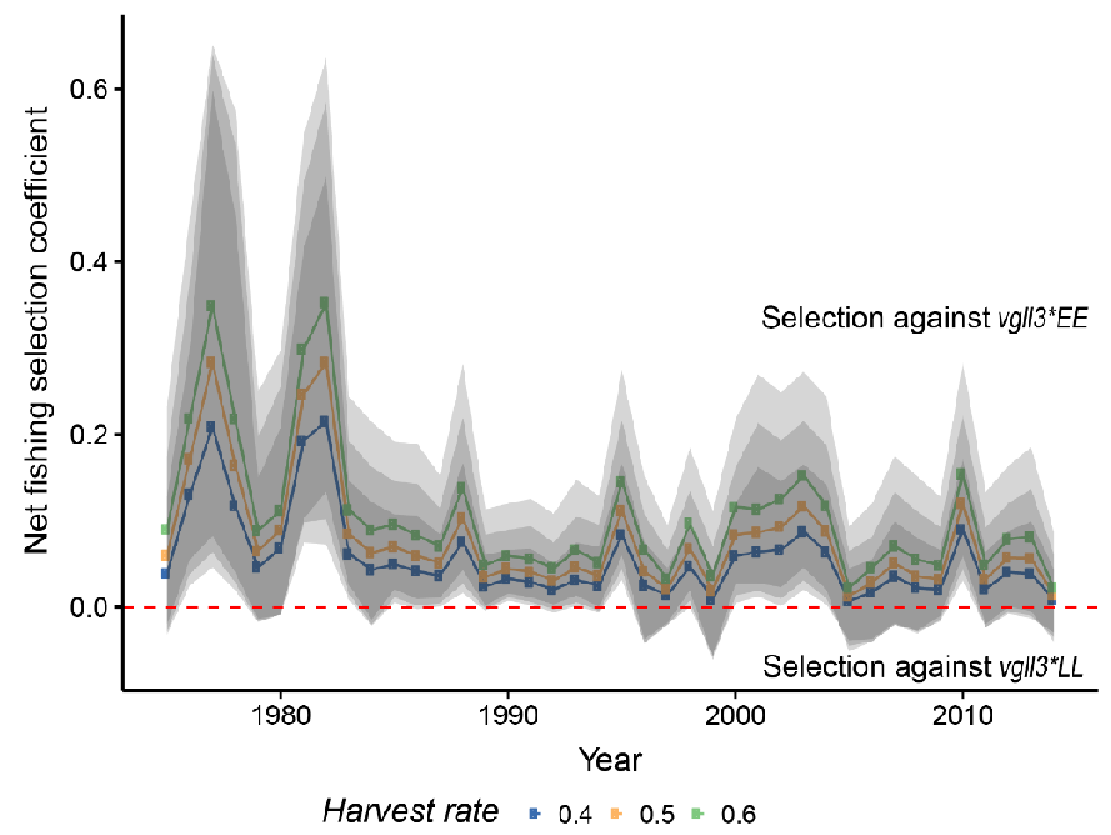

151 Figure 3: Predicted variation of net fishing induced selection over 40 years in 152 Tenojoki as a function of harvest rate. The selection coefficient corresponds to the relative 
153

154

155

156

157

158

159

160

161

162

163

164

165

166

167

168

169

170

171

172

173

174

175

176

177

178

179

180

181

182

183

184

185

difference in survival between $v g l l 3^{*} E E$ and $v g l l 3 * L L$. Calculations are based on capture probabilities (with harvest rates ranging from 40-60\% of returning individuals) as a function of length, using sonar data in 2018 and 2019. It accounts for the sex-specific vgll3 association with length during the study period, temporal variation in sex-ratio and relative use of the weir, driftnet and gillnet (see methods). Shaded area represents $95 \%$ confidence intervals based on 6000 iterations.

We provide an example of fisheries induced evolution, where both direct and indirect fishing effects contributed. Minimizing fisheries induced evolution is generally recommended as adaptation of populations to fishing can hinder adaptation to their natural environment and be costly in the long run ${ }^{21-23}$. However, strong socio-economic pressures may limit opportunities for dramatic changes in fishing regulations to alter harvest rates to minimise fisheries induced evolution ${ }^{23}$. In the Teno river valley for instance, salmon fishing is both a key source of income for locals through fishing tourism, as well as the foundation of the indigenous Sámi culture and identity, and restrictive fisheries regulations can be controversial ${ }^{24}$. The varying selectivity of different net types may provide a means to manage the selection pressures exerted by fishing on the different ages at maturity and genotypes e.g. by regulating the relative use of the different fishing gears. Collection of additional data (e.g. additional years of genetic stock assignment and sonar counting) and the development of models coupling population dynamics and evolutionary genetics would be required prior to implementation. Such models would also help in identifying constant selective pressures that couldn't be detected using regressions and in determining the impact of fisheries induced selection on population growth rate. Commercial harvesting of an important salmon prey species, capelin, indirectly induced evolution of Atlantic salmon age at maturity toward younger, smaller individuals. Several studies have noted the potential for a fishery to induce ecological effects beyond the target species ${ }^{25}$. However, evolutionary effects have not been demonstrated earlier ${ }^{5,13}$. This case also has important implications of a more applied nature. About $90 \%$ of the forage fish catch are used for fish oil and fishmeal to feed farmed animals ${ }^{6}$ and salmonid fish aquaculture is the $4^{\text {th }}$ highest consumer ${ }^{26}$. For instance in $2012,75,800$ tons of capelin was used in Norwegian aquaculture salmon feed (representing $15 \%$ of the marine ingredients ${ }^{27}$ ). Our results therefore identify yet another indirect path by which Atlantic salmon aquaculture can negatively affect wild populations of the same species and emphasize the importance of identifying alternative, sustainable, protein sources for the aquaculture industry.

\section{Material \& methods}

\section{Sampling, genotyping and population assignment}


186 The Teno river (Deatnu in Sámi, Tana in Norwegian), located at the border between Finland and

187 Norway and draining into the Barents Sea $\left(68-70^{\circ} \mathrm{N}, 25-27^{\circ} \mathrm{E}\right)$, hosts large and diverse Atlantic 188 salmon populations ${ }^{20,28}$. Scales of more than 150000 salmon caught in different parts of the Teno 189 river system have been collected since 1971, along with individual information including sex, 190 length, weight, sea age at maturity, fishing gear (driftnet, weir, gillnet or rod) and fishing location. 191 Of these, around 113000 were collected in the mainstem, the focal region of this study. Teno 192 salmon can remain from 2 to 8 years in fresh water before migrating to the sea. They then stay in 193 the marine environment, where most growth occurs, for mostly 1 to 3 years, occasionally 4 or 5 , 194 before maturing and returning to spawn ${ }^{20}$. Details about sample collection, DNA extraction, 195 genotyping (at 191 SNPs, including vgll3, and a sex marker, sdy ${ }^{29}$ ) and population assignment are 196 described in ${ }^{3}$. Briefly, a total of 1319 individuals genetically assigned to the Tenojoki population in 197 the middle reaches of the mainstem of the river system (hereafter Tenojoki) in ${ }^{3}$ were used in this 198 study, resulting in an average annual sample size of 33 individuals per year between 1975 and 2014.

\section{Environmental variables}

200 Evolution of age at maturity can be driven by factors occurring either in the freshwater or marine 201 phases of salmon life history. Changes in pelagic communities of the Barents Sea may affect 202 Atlantic salmon survival and life history traits during the marine feeding phase e.g. 30,31. The stock 203 biomass of important salmon prey species (capelin, herring and krill) have been monitored for 204 decades in the Barents Sea. Data about krill biomass $(1980-2013)$ were taken from ${ }^{32,33}$. Capelin 205 biomass estimated from acoustic survey and the landed capelin catches were derived from ${ }^{34}$ for 2061973 - 2013. Herring dynamics has been estimated using a virtual population analysis (VPA) over 207 the last decades ${ }^{35}$. Because herring migrate out of the Barents Sea once they mature, at the age of 208 3-5 years ${ }^{36}$, only the biomass of 1-2 year old herring were used here ${ }^{\text {e.g. } 17}$. These herring biomass 209 data were retrieved from ${ }^{35}$ for the 1973-1998 period. Herring biomass was calculated from the 210 number of 1-2 year old herring and the mean weight per age as reported in ${ }^{34}$ for $1988-2013$. The 211 Pearson correlation between biomasses from those two datasets of herring was 0.99 for the 212 overlapping period, despite values in ${ }^{34}$ being lower by a factor 1.46 . The data from ${ }^{35}$ were 213 standardised accordingly by dividing them by 1.46. Data about several species interacting with 214 salmon prey were also used in the multi-species model (see below). The annual biomass of cod (a 215 predator of forage fish) was derived from VPA analyses $\left({ }^{37}\right.$, table 3.24). Landed cod biomass was 216 also taken from ${ }^{37}$. Further, an index for mesozooplankton (a forage fish food source) corresponding 217 to the sum of Calanus biomass indices from different parts of the Barents Sea was used ${ }^{38}$. Marine 218 temperature has often been associated with growth, age at maturity or survival in Atlantic salmon 
219

220

221

222

223

224

225

226

227

228

229

230

231

232

233

234

235

236

237

238

239

240

241

242

243

244

245

246

247

39,e.g. 40 . The (monthly averaged) sea temperature in the Kola section measured in the upper 200 meters was therefore included (from Pinro.com, ${ }^{41}$ ).

Salmon fisheries targeting adult salmon returning from their marine migration occur both in the coastal areas around the outlet of the Teno River (i.e. the Finnmark region), and in the river itself using fishing gears with varying degrees of selectivity on size, and hence, age at maturity ${ }^{20,42}$. The total number of nets used to catch salmon in the Finnmark coastal region was calculated for each year using data from ${ }^{43}$ whereas the annual number of net fishing licenses, corrected for the number of fishing days allowed per week (which changed from four days to three days in 1980) was used as a surrogate for riverine net fishing effort. The corrected number of net fishing licenses had an among years Pearson's correlation of $0.75\left(\mathrm{t}_{34}=7.35\right.$, P-value $\left.<0.001\right)$ with the number of weirs in the Teno river, estimated from count data during the 1976-2014 time period and adjusted for the number of fishing days in a week (data from years 1980 to 1982 were missing for the latter).

A sonar count provided an estimate of the size distribution of ascending salmon, thus enabling an estimation of the size selectivity of riverine fishing methods via comparison with the size distribution of salmon caught with each fishing gear type during the fishing period in June and July. An ARIS explorer 1200 sonar unit (Sound Metrics Corp., Bellevue, Washington, USA) was placed c. $55 \mathrm{~km}$ upstream of the Teno river mouth in 2018 and 2019. Only individuals with a length greater than $43 \mathrm{~cm}$ were considered as salmon, because of the occurrence of other fish species which are mostly smaller than salmon. The number of upstream migrating salmon was calculated as the difference between the number of ascending and descending individuals. In 2019, the total number of salmon in the $136 \mathrm{~cm}$ size group was adjusted from minus one (meaning overall, one more fish in the size group descended than ascended), to zero. The length of salmon caught in 2018-2019 with driftnet, weir, gillnet and rod was recorded by fishers $(\mathrm{N}=17,745,95$ and 219 in 2018 and $\mathrm{N}=45$, 717, 71 and 334 in 2019; respectively). Salmon lengths from sonar data and catches were grouped in $2 \mathrm{~cm}$ bins from $44 \mathrm{~cm}$ to $151 \mathrm{~cm}$ and used for estimating selectivity of different fishing methods (see below). A small number of individuals that were estimated to be larger than $150 \mathrm{~cm}$ in the 2018 sonar count $(\mathrm{N}=17)$ were grouped with the last size class bin. One $136 \mathrm{~cm}$ rod-caught individual was moved to the $134-135 \mathrm{~cm}$ bin in 2019 as no individual of that size class was observed by sonar in that year. 


\section{Statistical analyses}

\section{Driver of vgll3 evolution}

250 To identify the environmental drivers of $v g l l 3$ evolution, the proportion of $v g l l 3 * L$ alleles $(0,0.5$ or

251 1) in an individual was regressed using a Generalized Linear Model (GLM) with the quasi-binomial 252 family e.g. 44 . The prey biomass (krill, capelin and herring) and sea temperature were averaged over 253 the year(s) each individual spent in the marine environment, which was inferred from scale growth 254 ring information. The logarithm of each prey's biomass was included as an independent variable to 255 allow non-linear associations with the vgll3 proportions and a better correspondence with the 256 multispecies model described below. The mean sea temperature, the number of coastal fishing nets, 257 the number of tourist rod-fishing licenses $\left(\right.$ days $^{-1}$ ) and net-fishing licenses in the Teno river, 258 corrected for the number of permitted weekly fishing days, were also included as predictors. Model 259 selection was performed with a hypothesis testing approach using backward selection with $\mathrm{F}$ tests. 260 Statistical tests throughout the study were two-tailed, using an alpha value 0.05 and were performed 261 using $\mathrm{R}^{45}$. The AICc of all possible models were also calculated along with the relative importance 262 of the different variables, using the MuMIn package ${ }^{46}$. Model averaging with the classical method $263{ }^{47}$ was performed on the subset of models accumulating an AICc weight of 0.95. For graphical 264 representations, fitted allele frequencies were averaged per hatching year and bias-corrected and 265 accelerated bootstrap intervals (BCa) were calculated with the BOOT package ${ }^{48}$.

266 Co-occurrence of temporal trends in both the dependent and independent variables may lead to 267 spurious detection of environmental effects. To account for this, de-trending of the data was 268 achieved as advised in ${ }^{49}$ by conducting residual regression on the original data ${ }^{15}$. To do this, the 269 independent variables included in the GLM were regressed in a linear model against years and 270 squared years to estimate linear or quadratic trends, respectively. The squared year term was then 271 removed from the model if not significant (assessed by F tests). Residuals of these models then 272 replaced the original independent variables. The GLM described above was re-run by including an 273 additional spawning year effect to remove temporal trends in vgll3 allele frequency. The spawning 274 year was kept as a covariate during the model selection process even if it was not significant.

\section{Drivers of sex-ratio changes}

276 To identify variables modifying the sex-ratio of returning adults in Tenojoki, a binomial GLM was 277 performed by coding the sex of individuals as 0 (male) or 1 (female). The log biomass of prey 278 species, sea temperature, number of coastal fishing nets and number of riverine net and rod (days ${ }^{-1}$ ) 279 fishing licences were included as independent variables as previously. Model selection was realized 
280

281

282

283

284

285

286

287

288

289

290

291

292

293

294

295

296

297

298

299

300

301

302

303

304

305

306

307

308

309

310

using the Likelihood ratio test (backward variable selection) and the AICc criterion. This analysis was repeated by using the residual regression method to account for potential co-occurrences of temporal trends in the dependent and independent variables, as described above.

\section{Drivers of changes in age at maturity}

Age at maturity of individual salmon was regressed in a multinomial model using all the independent variables described above ( $\mathrm{R}$ package nnet ${ }^{50}$ ). Additionally, vgll3 genotypes and sex were included in the model in a two-way interaction, due to known vgll3 sex-specific effects ${ }^{4}$. Two individuals that matured after five years at sea were considered as individuals having matured after four years at sea. Variable selection with F tests and AICc model averaging were also performed. This analysis was repeated by using the residual regression method.

\section{Indirect evolutionary effects}

The indirect effect of capelin harvesting on vgll3 evolution was estimated using the Monte Carlo Method for Assessing Mediation (MCMAM) ${ }^{51}$ with capelin $(\log )$ biomass as the mediator. The effect of $(\log )$ capelin biomass on $v g l l 3 * L$ odd-ratio was obtained with the quasi-binomial GLM model described above (after backward selection, including the capelin, herring, krill and riverine net fishing licenses variables). The estimated parameter and standard error were used to generate 3000 samples drawn from a normal distribution. The posterior distribution of the direct effect of harvest rate on the $(\log )$ capelin biomass was obtained with the multispecies Gompertz model described below (3 000 MCMC samples, Extended Data Fig. 4, equation 1). The indirect effect of capelin harvest on $\operatorname{vgll}{ }^{*} L$ odd-ratio corresponds to the product of the two sets of samples (i.e. effect of capelin fishing on ( $\log$ ) capelin $\mathrm{x}$ effect of (log) capelin on vgll3 odds) and thus accounts for uncertainty in both estimates. The indirect effect was also evaluated graphically to account for more than one interaction, by:

1) Using the multispecies Gompertz model to predict the biomass dynamics of capelin, herring and krill when capelin harvesting is set to zero from the first year of the time series;

2) Using the newly generated data (posterior median) to predict the vgll3 allele frequency variation using the quasi-binomial GLM obtained with backward selection

3) Plotting the temporal allele frequency variation with Bca confidence intervals

This analysis accounted for the effect of capelin harvesting on the biomass of all the Gompertz model species but didn't account for vgll3 evolutionary dynamics (i.e. the propagation of the indirect negative effect from one generation to the next). 


\section{Multispecies Gompertz model}

312 Fish populations in the Barents Sea have experienced large variation in their abundance over the 313 last 40 years due to fishing and predation ${ }^{\text {e.g. } 16}$. Consequently, important indirect effects on Atlantic 314 salmon age at maturity variation/evolution may be expected. In order to estimate potential indirect 315 effects, the Barents Sea species interactions, the effect of temperature, density dependence and of 316 cod and capelin fishery were estimated using the multispecies Bayesian Gompertz model developed 317 in ${ }^{17}$. To summarize, on the $\log$ scale, the process equations were:

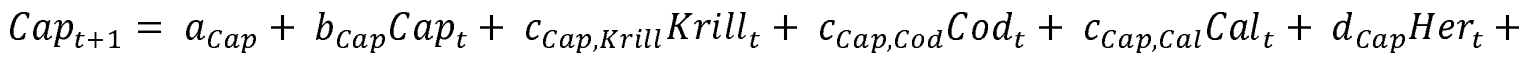

$$
\begin{aligned}
& e_{C a p} T_{t}+f_{C a p} C_{C a p, t}+P E_{c a p, t}(\text { Equation 1) } \\
& \text { Krill }_{t+1}=a_{\text {Krill }}+b_{\text {Krill }} \text { Krill }_{t}+c_{\text {Krill,Cap }} \text { Cap }_{t}+d_{\text {Krill }} \text { Her }_{t}+e_{\text {Krill }} T_{t}+P E_{\text {Krill }, t} \\
& \operatorname{Cod}_{t+1}=a_{C o d}+b_{C o d} \operatorname{Cod}_{t}+c_{C o d, C a p} C a p_{t}+d_{C o d} H_{e r}+e_{C o d} T_{t}+f_{C o d} C_{C o d, t}+P E_{C o d, t} \\
& \mathrm{Cal}_{t+1}=a_{C a l}+b_{C a l} \mathrm{Cal}_{t}+c_{C a l, C a p} \mathrm{Cap}_{t}+d_{C a l} \mathrm{Her}_{t}+e_{C a l} T_{t}+\mathrm{PE}_{\text {Cal, } t}
\end{aligned}
$$

With Cap ${ }_{t}, \mathrm{Krill}_{\mathrm{t}}, \mathrm{Cod}_{\mathrm{t}}, \mathrm{Cal}_{\mathrm{t}}$ and $\mathrm{Her}_{\mathrm{t}}$ being the log biomass of capelin, krill, cod, Calanus and herring, respectively, in year $t, \mathrm{~T}_{\mathrm{t}}$ the annual sea temperature in the Kola section of the Barents Sea at $0-200 \mathrm{~m}$ depth (along the $33^{\circ} 30^{\prime} \mathrm{E}$ meridian from $70^{\circ} 30^{\prime}$ to $72^{\circ} 30^{\prime} \mathrm{N}$ ) in year $t$ and $\mathrm{C}_{\text {Cap,t }}$ and $\mathrm{C}_{\text {Cod,t }}$ the capelin and cod harvest rates, respectively, in year $t$ (catch/annual biomass). The coefficients $a$, $b$ and $c$ represent the productivity, density dependence and interactions among modelled species, respectively. The coefficient $d$ represents the interactions with herring, $e$ the temperature effects, $f$

327 the fishing effects and PEs the multivariate-normal-distributed process errors. The prior 328 distributions in the model were identical to ${ }^{17}$ (e.g. uniform prior distributions from -10 to 10 for all process parameters $a, b, c, d, e$ and $f$ ).

330 Posterior distributions were approximated using MCMC methods with the Just Another Gibbs 331 Sampler software JAGS ${ }^{52}$. The model was run for 1410000 iterations including a burn-in length 332 of 710 000. One iteration out of 1000 was kept and 3 chains were run. Convergence was assessed 333 using the Gelman and Rubin's convergence diagnostic and a Potential Scale Reduction Factor 334 (PSRF) threshold of $1.15^{53}$. The model posterior medians were substituted to the original data for 335 other analyses, in order to replace missing values in krill from 1973 to 1979 and employ common 336 data between the different models used for indirect effect evaluations. 
337

338

339

340

341

342

343

344

345

346

347

348

349

350

351

352

353

354

355

356

357

358

359

360

361

362

363

364

365

366

367

\section{Net fishing size selectivity}

Teno local net fishing encompass three fishing methods with different size selectivities ${ }^{20}$. To better understand the potential effect of net fishing on vgll3 allele frequency changes, fishing size selectivity in Tenojoki salmon was estimated by using sonar count data and the length distribution of catches per fishing gear in 2018 and 2019 (including driftnet, weir, gillnet and rod). The number of salmon caught per $2 \mathrm{~cm}$. length class $(l$, from 44 to $151 \mathrm{~cm})$ was analyzed for each gear $(\mathrm{g})$ with a Generalized Additive Model (GAM) using the beta-binomial family to account for overdispersion. Length of each salmon $(L)$ was included as a predictor, in a regression spline $\left(s_{g}\right)$ :

$$
C_{l, g, y}=\alpha_{y}+s_{g}(L)
$$

With $C_{l, g, y}$ the mean proportion of salmon caught per $2 \mathrm{~cm}$. length class $(l)$ in year $(y)$ with the fishing gear $(\mathrm{g})$ and, $\alpha_{y}$ the annual intercepts with priors following a normal distribution (mean $=0$, $\mathrm{sd}=10$ ). The dispersion parameters of the beta distribution (i.e. addition of both shape parameters) followed a prior uniform distribution between 2.5 and 5000 for each gear. The priors, code and associated data for the smooth part of the model were generated with the R package $m g c v$ (see ${ }^{54}$ for more information). The model was run in JAGS ${ }^{52}$ (R package runjags ${ }^{55}$ ) for 7600000 iterations including a burn-in period of 4000 000. Two chains were run and one iteration out of 1200 was kept. Convergence was checked using the Gelman and Rubin's convergence diagnostic ${ }^{53}$ and inspection of trace plots. $C 2_{i, l, g}$, used below, corresponds to the mean capture probability of the gear $(g)$ per $2 \mathrm{~cm}$. length class $(l)$ calculated at each iteration $(i)$ from the model estimates, and divided by $\sum_{l=1}^{54} C 2_{i, l, g}$ so that it sums to one over the different length groups.

\section{Temporal variation in net fishing selection on vgll3}

The effect of riverine net fishing on vgll3 allele frequency depends on the size selectivity of the fishing gears, calculated above, the harvest rate per fishing gear and distribution of vgll3 genotypes according to salmon size. The number of salmon assigned to the mainstem population was modelled with a GAM using the negative binomial family, to allow for over-dispersion. Length was included as an independent variable inside annual random smooths, to account for changes in age at maturity over time. All GAM models in the following part of the method were run using the mgcv package ${ }^{56}$. To account for uncertainty, simulations from the posterior distribution of the fitted model were performed by using a Metropolis Hastings sampler ${ }^{57}$ (8 chains, 300000 iterations per chain, thinning of 400, burn-in of 1000). For each iteration $(i)$, the proportion of salmon in the different 2 $\mathrm{cm}$ length classes $\left(P l_{i, l, y}\right)$ was calculated for each year $(y)$ from the model predicted values. Those proportions were later used to estimate the gear specific fishing mortality to match the expected 
368

369

370

371

372

373

374

375

376

377

378

gear-specific annual harvest rates (see below). A model including annual random smooths per sex was also used to estimate $P l 2_{i, l, s, y}$, the proportion of salmon in the different $2 \mathrm{~cm}$ length class per sex and year at each iteration (i) (Metropolis Hastings sampling with 8 chains, 300000 iterations per chain, thinning of 400, burn-in of 1000).

The proportion of salmon caught by each fishing gear over the 40 -year period $\left(\operatorname{Pr}_{y, g}\right)$ was estimated from the annual catch weight, converted to an estimate of the individual number using the annual average weights of salmon caught in the mainstem with the different gears $(\mathrm{N}=84452)$. The total harvest rate $\left(H R_{\text {tot }}\right)$ was fixed at either $40 \%, 50 \%$ or $60 \%$ (as riverine fishing mortality of up to $69 \%$ on tagged salmon has been reported $\left.{ }^{58}\right)$, only the gear specific harvest rate $\left(H R_{\text {exp }}, g\right)$ being allowed to vary over time according to the previously calculated proportions $\left(P r_{y, g}\right)$ :

$$
H R_{\exp _{y, g}}=P r_{y, g} H R_{t o t}
$$

Given that fishing with the different gears occurs simultaneously, the instantaneous fishing mortality needed to be estimated according to the expected harvest rate $\left(H R_{e x p_{y, g}}\right)$ and gear size selectivity $\left(C 2_{i, l, g}\right)$. A modified version of the Baranov's catch equation was used to calculate the harvest rates $\left(H R_{i, y, g}\right)$ of the gear $(g)$ in year $(y)$ at each iteration $(i)$ :

$$
H R_{i, y, g}=\sum_{l=1}^{54}\left(1-e^{-\left(\sum_{g=1}^{4} F_{i, l, y, g}\right)}\right) \frac{F_{i, l, y, g}}{\sum_{g=1}^{4} F_{i, l, y, g}} \operatorname{Plcorr}_{i, l, y}
$$

With $\operatorname{Plcorr}_{i, l, y}$ the proportion of salmon in the $2 \mathrm{~cm}$. length class $(l)$ in year $(y)$ at iteration $(i)$ before fishing $\left(\right.$ Plcorr $_{i, l, y}=\frac{P l_{i, l, y}}{e^{-\left(\sum_{g=1}^{4} F_{i, l, y, g}\right)}}$, modified to sum to one over the length classes). $F_{i, l, y, g}$ is the instantaneous fishing mortality for the length class $(l)$, gear $(g)$ and year $(y)$ calculated at each iteration from the mean capture probabilities $C 2_{i, l, g}$ :

$$
F_{i, l, y, g}=-\log \left(1-C 2_{i, l, g} \beta_{i, y, g}\right)
$$

$\beta_{i, y, g}$ was determined by minimizing $\left.D=\sum_{i}^{4}\left(H R_{i, y, g}-H R_{\text {exp }}\right)_{y}\right)^{2}$ using the $\mathrm{R}$ optim function from the stats $r$ package with the "L-BFGS-B" method ${ }^{59}$ and a lower bound of 0.01 . The NelderMead method was used if convergence was not achieved with the "L-BFGS-B" method (12 iterations), while ensuring that $\beta_{i, y, g}$ was positive (e.g. by squaring it). The maximum instantaneous fishing mortality was capped at 2.30, allowing a maximum harvest rate per fishing gear of 0.95 . 
392 This was used to avoid unrealistic correction, at some iterations, of the length-frequency 393 distribution when calculating Plcorr $_{i, l, y}$.

394 The exploitation rates per year $(y)$, vgll3 genotype $(v)$ and net fishing gear $(g)$ were then calculated 395 at each iteration $(i)$ as follows:

$$
E R_{i, y, v, g, s}=\sum_{l=1}^{54}\left(1-e^{-\left(\sum_{g=1}^{4} F_{i, l, y, g}\right)}\right) \frac{F_{i, l, y, g}}{\sum_{g=1}^{4} F_{i, l, y, g}} \operatorname{Plg}_{i, \mathrm{l}, \mathrm{y}, \mathrm{s}, \mathrm{v}}
$$

With $\operatorname{Plg}_{i, 1, \mathrm{y}, \mathrm{s}, \mathrm{v}}$ the proportion of individuals of $\operatorname{sex}(s)$ in the $2 \mathrm{~cm}$. length class $(l)$ in year $(y)$ and 398 with the vgll3 genotype $(v)$, calculated at each iteration $(i)$ as follow:

$$
\operatorname{Plg}_{i, \mathrm{l}, \mathrm{y}, \mathrm{s}, \mathrm{v}}=\frac{P l 2 \operatorname{corr}_{i, l, y, s}}{\sum_{l=1}^{54} P l 2 \operatorname{corr}_{i, l, y, s}} \operatorname{Pvgll}_{i, l, s}
$$

And modified to sum to one over the length class. $P l 2 \operatorname{corr}_{i, l, s, y}$ is the proportion of individuals of 400 sex $(s)$ in the length class $(l)$ in year $(y)$ before fishing, calculated at each iteration $(i)$ from the 401 length distribution previously estimated with the GAM:

$$
P l 2 \operatorname{corr}_{i, l, y, s}=\frac{P l 2_{i, l, y, s}}{e^{-\left(\sum_{g=1}^{4} F_{i, l, y, g}\right)}}
$$

$P v g l l 3_{i, l, s}$ corresponds to the probability of the different $v g l l 3$ genotypes for a salmon in the $2 \mathrm{~cm}$ length class $(l)$ and sex $(s)$. This probability was estimated with a multinomial GAM including the $2 \mathrm{~cm}$ length classes in a regression spline for each sex and genotype. Posterior simulations (1 chain, 6000000 iterations, thinning of 1000, burn-in of 1000) were performed using the Metropolis Hastings sampler. Alternative models allowing the smooth parameter to change per sex and decades in one or both of the modelled genotypes were also run. There was no evidence for changes in the 408 length-specific probability of the different $v$ gll3 genotypes over time (model fitted with maximum likelihood, $\triangle \mathrm{AIC}>8$ and LRT test $\mathrm{P}$-value $=0.096$ with the best competing model).

410 Finally, the mortality per genotype $(v)$ and year $(y)$ for the 3 net fishing methods in the population 411 corresponded to:

$$
M_{i, y, v}=\sum_{g=1}^{3} E R_{i, y, v, g, s=f e m a l e} S R_{-} \operatorname{corr}_{i, y, v}+\sum_{g=1}^{3} E R_{i, y, v, g, s=m a l e}\left(1-S R_{-} \operatorname{corr}_{i, y, v}\right)
$$


413 The mortality per genotype accounts for temporal variation in the proportion of females per 414 genotype before fishing $\left(S R_{-} \operatorname{corr}_{i, y, v}\right)$. Temporal variation in the female salmon proportion was 415 analysed with a quasi-binomial GAM, including year as an independent variable inside a cubic 416 regression spline for each genotype and adding an extra penalty to each term. To account for 417 uncertainty, simulations from the posterior distribution of the fitted models were performed by 418 randomly drawing 6000 values from a multivariate normal distribution with the mean vector and the 419 covariance matrix equal to the model estimates. The GAM fitted values were calculated at each 420 iteration to obtain $S R_{i, y, v}$. The proportion of females before fishing corresponded to:

$$
S R_{-} \operatorname{corr}_{i, y, v}=\frac{\left(1-\sum_{g=1}^{4} E R_{i, y, v, g, s=m a l e}\right) S R_{i, y, v}}{1-\sum_{g=1}^{4} E R_{i, y, v, g, s=f e m a l e}\left(1-S R_{i, y, v}\right)-\sum_{g=1}^{4} E R_{i, y, v, g, s=\text { male }} S R_{i, y, v}}
$$

421 The survival of the different genotypes could then be calculated: $W_{i, y, v}=1-M_{i, y, v}$. The annual 422 fishing selection against the less fitted homozygote $\left(S_{y}\right)$ was calculated as follows:

$$
S_{i, y}=1-\min \left(W_{i, y, 1}, W_{i, y, 3}\right) / \max \left(W_{i, y, 1}, W_{i, y, 3}\right)
$$

423 The sign of $S_{y}$ was changed according to $\max \left(W_{i, y, 1}, W_{i, y, 3}\right) ; S_{y}$ is positive when $W_{i, y, 1},<W_{i, y, 3}$ 424 and negative otherwise. Calculations of variation in selection and relative mortality assume 425 temporally constant gear selectivity. The total harvest rate is also assumed constant over time and 426 was included to assess whether the fishing pressure in the Teno river is strong enough to induce significant selection.

428 Effect of changes in net fishing selection on $v g l l 3$, at a constant harvest rate of 0.5 , were 429 investigated in a post-hoc analysis by included $S_{i, y}$ in the normal and de-trended quasi-binomial GLM previously described.

$431 \quad$ Data and code availability:

432 The datasets used in the current study will be uploaded to a public data repository upon acceptance.

433 Code availability:

434 The personal custom codes used in the current study will be uploaded to a public data repository 435 upon acceptance.

436 Availability of biological material:

437 All unique biological materials used are available from the authors 


\section{References}

440 1. Merilä, J. \& Hendry, A. P. Climate change, adaptation, and phenotypic plasticity: The $441 \quad$ problem and the evidence. Evol. Appl. 7, 1-14 (2014).

442 2. Forseth, T. et al. The major threats to Atlantic salmon in Norway. ICES J. Mar. Sci. 74, $443 \quad 1496-1513$ (2017).

3. Czorlich, Y., Aykanat, T., Erkinaro, J., Orell, P. \& Primmer, C. R. Rapid sex-specific evolution of age at maturity is shaped by genetic architecture in Atlantic salmon. Nat. Ecol. Evol. 2, 1800-1807 (2018).

4. Barson, N. J. et al. Sex-dependent dominance at a single locus maintains variation in age at maturity in salmon. Nature 528, 405-408 (2015).

5. Heino, M., Díaz Pauli, B. \& Dieckmann, U. Fisheries-Induced Evolution. Annu. Rev. Ecol. Evol. Syst. 46, 461-480 (2015).

6. Pikitch, E. K. et al. The global contribution of forage fish to marine fisheries and ecosystems. Fish Fish. 15, 43-64 (2014).

7. Gienapp, P., Teplitsky, C., Alho, J. S., Mills, J. A. \& Merilä, J. Climate change and evolution: Disentangling environmental and genetic responses. Mol. Ecol. 17, 167-178 (2008).

8. Walsh, M. R. The evolutionary consequences of indirect effects. Trends Ecol. Evol. 28, 23-

9. Tylianakis, J. M., Didham, R. K., Bascompte, J. \& Wardle, D. A. Global change and species interactions in terrestrial ecosystems. Ecology Letters 11, 1351-1363 (2008).

460

10. Crowder, L. B. et al. The Impacts of Fisheries on Marine Ecosystems and the Transition to Ecosystem-Based Management. Annu. Rev. Ecol. Evol. Syst. 39, 259-278 (2008).

462

463

464

465

466

467

468

11. Kuparinen, A. \& Merilä, J. Detecting and managing fisheries-induced evolution. Trends Ecol. Evol. 22, 652-659 (2007).

12. Jennings, S. \& Kaiser, M. J. The effects of fishing on marine ecosystems. (Academic Press, 1998).

13. Hutchings, J. A. \& Kuparinen, A. Implications of fisheries-induced evolution for population recovery: Refocusing the science and refining its communication. Fish Fish. 21, 453-464 (2020).

14. Mobley, K. B. et al. Home ground advantage: Local Atlantic salmon have higher reproductive fitness than dispersers in the wild. Sci. Adv. 5, eaav1112 (2019).

15. Graham, M. H. Confronting Multicollinearity in Ecological Multiple Regression. Ecol. Soc. Am. 84, 2809-2815 (2003).

16. Hjermann, D. Ø., Ottersen, G. \& Stenseth, N. C. Competition among fishermen and fish causes the collapse of Barents Sea capelin. Proc. Natl. Acad. Sci. U. S. A. 101, 11679-11684 (2004). 
476 17. Langangen, Ø. et al. Cascading effects of mass mortality events in Arctic marine communities. Glob. Chang. Biol. 23, 283-292 (2017).

478

479

480

481

482

483

484

485

486

487

488

489

490

491

492

493

494

495

496

497

498

499

500

501

502

503

504

505

506

507

508

509

510

511

512

18. Ricker, W. E. Changes in the average size and average age of Pacific salmon. Can. J. Fish. Aquat. Sci. 38, 1636-1656 (1981).

19. Kendall, N. W. \& Quinn, T. P. Quantifying and comparing size selectivity among Alaskan sockeye salmon fisheries. Ecol. Appl. 22, 804-816 (2012).

20. Erkinaro, J. et al. Life history variation across four decades in a diverse population complex of Atlantic salmon in a large subarctic river. Can. J. Fish. Aquat. Sci. 76, 42-55 (2019).

21. Heino, M. et al. Can fisheries-induced evolution shift reference points for fisheries management? ICES J. Mar. Sci. 70, 707-721 (2013).

22. Walsh, M. R., Munch, S. B., Chiba, S. \& Conover, D. O. Maladaptive changes in multiple traits caused by fishing: impediments to population recovery. Ecol. Lett. 9, 142-148 (2006).

23. Hard, J. J. et al. Evolutionary consequences of fishing and their implications for salmon. Evol. Appl. 1, 388-408 (2008).

24. Hiedanpää, J., Saijets, J., Jounela, P., Jokinen, M. \& Sarkki, S. Beliefs in Conflict: The Management of Teno Atlantic Salmon in the Sámi Homeland in Finland. Environ. Manage. 66, 1039-1058 (2020).

25. Naylor, R. L. et al. Effect of aquaculture on world fish supplies. Nature 405, 1017-1024 (2000).

26. Froehlich, H. E., Jacobsen, N. S., Essington, T. E., Clavelle, T. \& Halpern, B. S. Avoiding the ecological limits of forage fish for fed aquaculture. Nat. Sustain. 1, 298-303 (2018).

27. Ytrestøyl, T., Aas, T. S. \& Åsgård, T. Utilisation of feed resources in production of Atlantic salmon (Salmo salar) in Norway. Aquaculture 448, 365-374 (2015)

28. Vähä, J.-P., Erkinaro, J., Falkegård, M., Orell, P. \& Niemelä, E. Genetic stock identification of Atlantic salmon and its evaluation in a large population complex. Can. J. Fish. Aquat. Sci. 74, 327-338 (2016).

29. Aykanat, T., Pritchard, V. L., Lindqvist, M. \& Primmer, C. R. From population genomics to conservation and management: a workflow for targeted analysis of markers identified using genome-wide approaches in Atlantic salmon. J. Fish Biol. 89, 2658-2679 (2016).

30. Friedland, K. D. et al. The recruitment of Atlantic salmon in Europe. ICES J. Mar. Sci. 66, 289-304 (2009).

31. Aykanat, T. et al. Life-history genomic regions explain differences in Atlantic salmon marine diet specialization. J. Anim. Ecol. 89, 2677-2691 (2020).

32. Eriksen, E. \& Dalpadado, P. Long-term changes in Krill biomass and distribution in the Barents Sea: Are the changes mainly related to capelin stock size and temperature conditions? Polar Biol. 34, 1399-1409 (2011).

33. ICES. Report of the Working Group on the Integrated Assessments of the Barents Sea. ICES 
CM 2017/SSGIEA:04. 186 pp. (2017).

514

515

516

517

518

519

520

521

522

523

524

525

526

527

528

529

530

531

532

533

534

535

536

537

538

539

540

541

542

543

544

545

546

547

548

549

34. ICES. Report of the Arctic Fisheries Working Group ( AFWG). ICES CM 2015/ACOM:05. 639 pp. (2015).

35. Toresen, R. \& Østvedt, O. J. Variation in abundance of Norwegian spring-spawning herring (Clupea harengus, Clupeidae) throughout the 20th century and the influence of climatic fluctuations. Fish Fish. 85, 385-391 (2000).

36. Krysov, A. \& Røttingen, I. Herring. in The Barents Sea: ecosystem, resources, management: Half a century of Russian-Norwegian cooperation (eds. T, J. \& VK, O.) 215-224 (Tapir Academic Press, 2011).

37. ICES. Report of the Arctic Fisheries Working Group (AFWG ). ICES CM 2016/ACOM:06. 621 pp. (2016).

38. Stige, L. C. et al. Spatiotemporal statistical analyses reveal predator-driven zooplankton fluctuations in the Barents Sea. Prog. Oceanogr. 120, 243-253 (2014).

39. Otero, J. et al. Contemporary ocean warming and freshwater conditions are related to later sea age at maturity in Atlantic salmon spawning in Norwegian rivers. Ecol. Evol. 2, 21922203 (2012).

40. Friedland, K. D. \& Todd, C. D. Changes in Northwest Atlantic Arctic and Subarctic conditions and the growth response of Atlantic salmon. Polar Biol. 35, 593-609 (2012).

41. Tereshchenko, V. V. Seasonal and year-to-year variations of temperature and salinity along the Kola meridian transect. CM 1996/C:11, pp 24. (1996).

42. Jensen, A. J. et al. Cessation of the Norwegian drift net fishery: changes observed in Norwegian and Russian populations of Atlantic salmon. ICES J. Mar. Sci. 56, 84-95 (1999).

43. Niemelä, E., Kalske, T. \& Hassinen, E. Numbers of fishing gears used in Kolarctic salmon project area, numbers of allowed sites for salmon fishing and numbers of salmon fishermen in Finnmark; development until the year 2013. (2013).

44. Schaid, D. J., Sinnwell, J. P. \& Jenkins, G. D. Regression Modeling of Allele Frequencies and Testing Hardy Weinberg Equilibrium. Hum Hered. 74, 71-82 (2012).

45. R Core Team. R: The R Project for Statistical Computing. (2017).

46. Barton, K. MuMIn: Multi-Model Inference. (2018).

47. Burnham, K. P. \& Anderson, D. R. Model selection and multimodel inference $\square$ : a practical information-theoretic approach. (Springer, 2003).

48. Canty, A. \& Ripley, B. D. boot: Bootstrap R (S-Plus) Functions. (2017).

49. Grosbois, V. et al. Assessing the impact of climate variation on survival in vertebrate populations. Biol. Rev. 83, 357-399 (2008).

50. Venables, W. N. \& Ripley, B. D. Modern Applied Statistics With S. (Springer, 2002).

51. MacKinnon, D. P., Lockwood, C. M. \& Williams, J. Confidence Limits for the Indirect Effect: Distribution of the Product and Resampling Methods. Multivariate Behav. Res. 39, 
37-67 (2004).

551

552

553

554

555

556

557

558

559

560

561

562

563

564

565

566

567

568

569

570

571

572

573

574

575

576

577

578

579

580

52. Plummer, M. JAGS Version 4.3.0 user manual. (2017).

53. Brooks, S. P. B. \& Gelman, A. G. General methods for monitoring convergence of iterative simulations. J. Comput. Graph. Stat. 7, 434-455 (1998).

54. Wood, S. N. Just Another Gibbs Additive Modeler $\square$ : Interfacing JAGS and mgcv. J. Stat. Softw. 75, 1-15 (2016).

55. Denwood, M. J. \{runjags $\}$ : An $\{\mathrm{R}\}$ Package Providing Interface Utilities, Model Templates, Parallel Computing Methods and Additional Distributions for \{MCMC $\}$ Models in \{JAGS . J. Stat. Softw. 71, 1-25 (2016).

56. Wood, S. N. Generalized additive models: an introduction with R. (CRC press, 2017).

57. Wood, S. N. Bayesian computation. in Core Statistics (Cambridge University Press, 2015).

58. Erkinaro, J., Økland, F., Moen, K. \& Niemelä, E. Return migration of the Atlantic salmon in the Tana River: Distribution and exploitation of radiotagged multi-sea-winter salmon. Boreal Environ. Res. 4, 115-124 (1999).

59. Byrd, R. H., Lu, P., Nocedal, J. \& Zhu, C. A Limited Memory Algorithm for Bound Constrained Optimization. SIAM J. Sci. Comput. 16, 1190-1208 (1995).

\section{Acknowledgements}

We thank fishers who collected scales and phenotypic information and people who organized and read the scales, especially Jorma Kuusela and Jari Haantie, as well as Jan-Peter Pohjola for sonar data reading, and Maija Länsman for compiling the detailed river catch and effort data. We also thank Eva Eriksen for providing corrected krill biomass data and Øystein Langangen for sharing codes and information on the multispecies Gompertz model. This project received funding from the Academy of Finland (projects No. 284941, 286334, 307593, 302873, 318939 and, 325964) as well as from the European Research Council (ERC) under the European Union's Horizon 2020 research and innovation programme (grant agreement No 742312). Part of YC salary was funded by the Norwegian Research Council (projects No. 275862 EcoEvoGene and 280308 SeaSalar).

\section{Author contributions}

J.E, and P.O. coordinated the collection of scale samples and river fisheries data; C.R.P., Y.C., T.A. and J.E. designed the study; Y.C realized the lab work, Y.C. analysed the data; Y.C., C.R.P. and T.A. wrote the manuscript and all authors contributed to its revision.

\section{Competing interests}

The authors declare no competing financial interests. 


\section{Materials \& Correspondence}

583 Correspondence and requests for materials should be addressed to C.R.P.

\section{Extended Data}

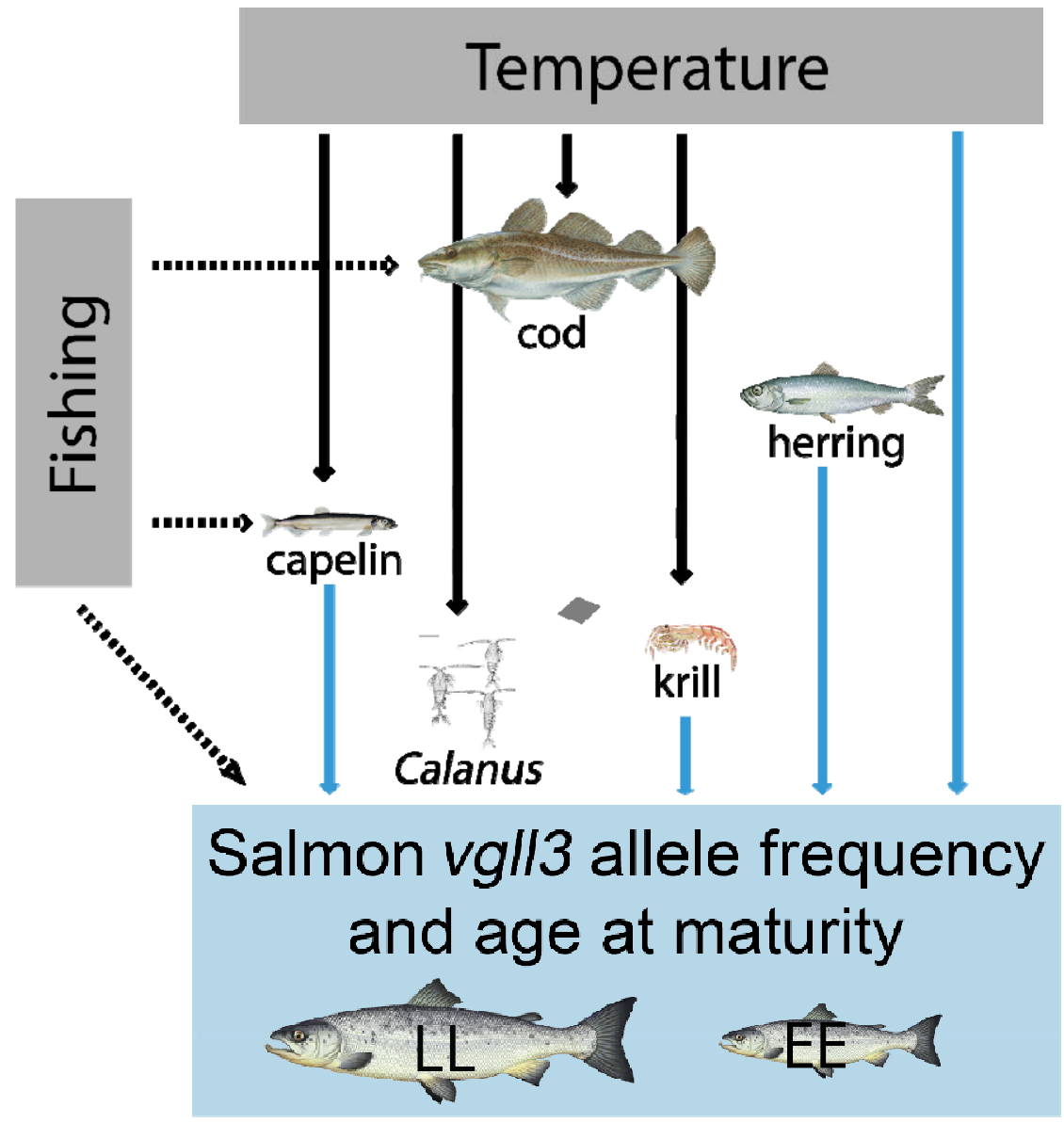

586 Extended Data Figure 1: Interactions in the Barents sea community as modelled by ${ }^{17}$ and links with Atlantic salmon age at maturity and vgll3 allele frequencies. 


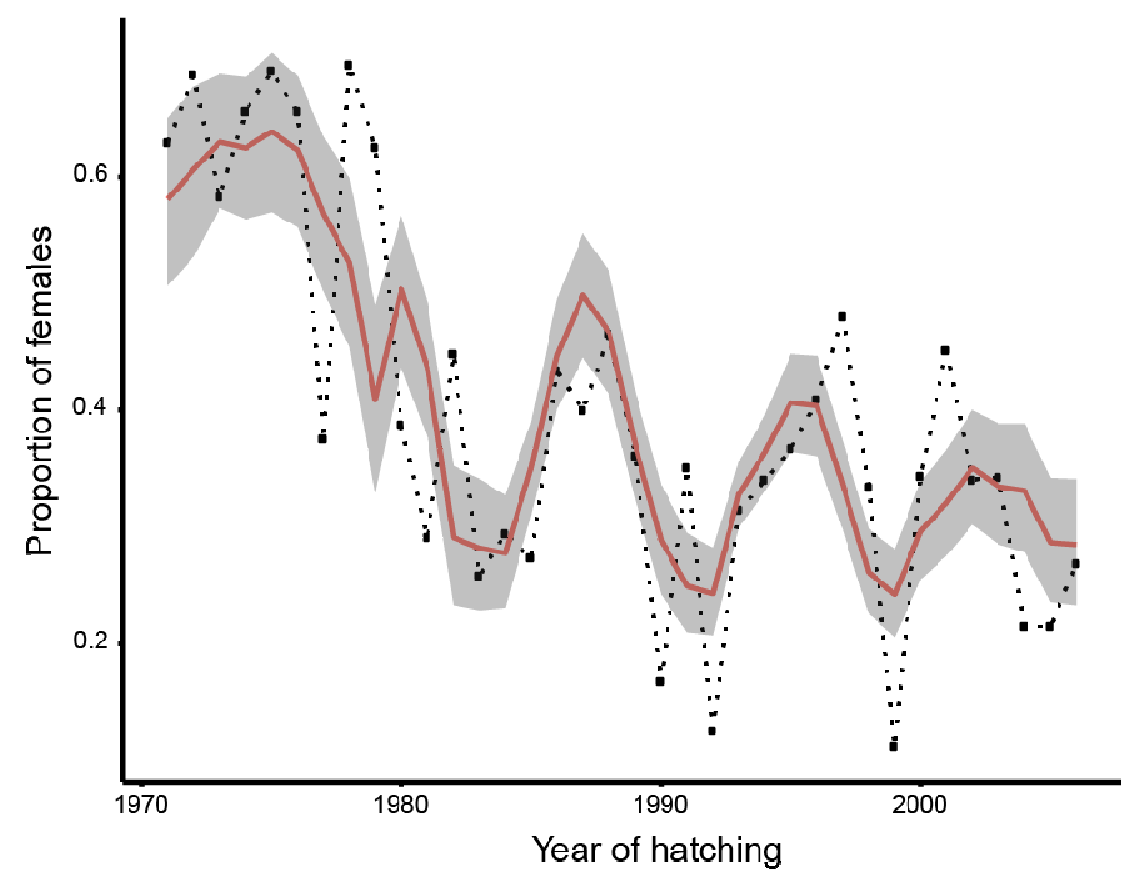

Extended Data Figure 2: Temporal changes in proportion of females over 36

hatching years. The black dotted line represents the observed data, the red line the female proportion averaged from individual fitted values. Confidence intervals are bias-corrected and accelerated (BCa) 95\% bootstrap interval based on 3000 replicates. 

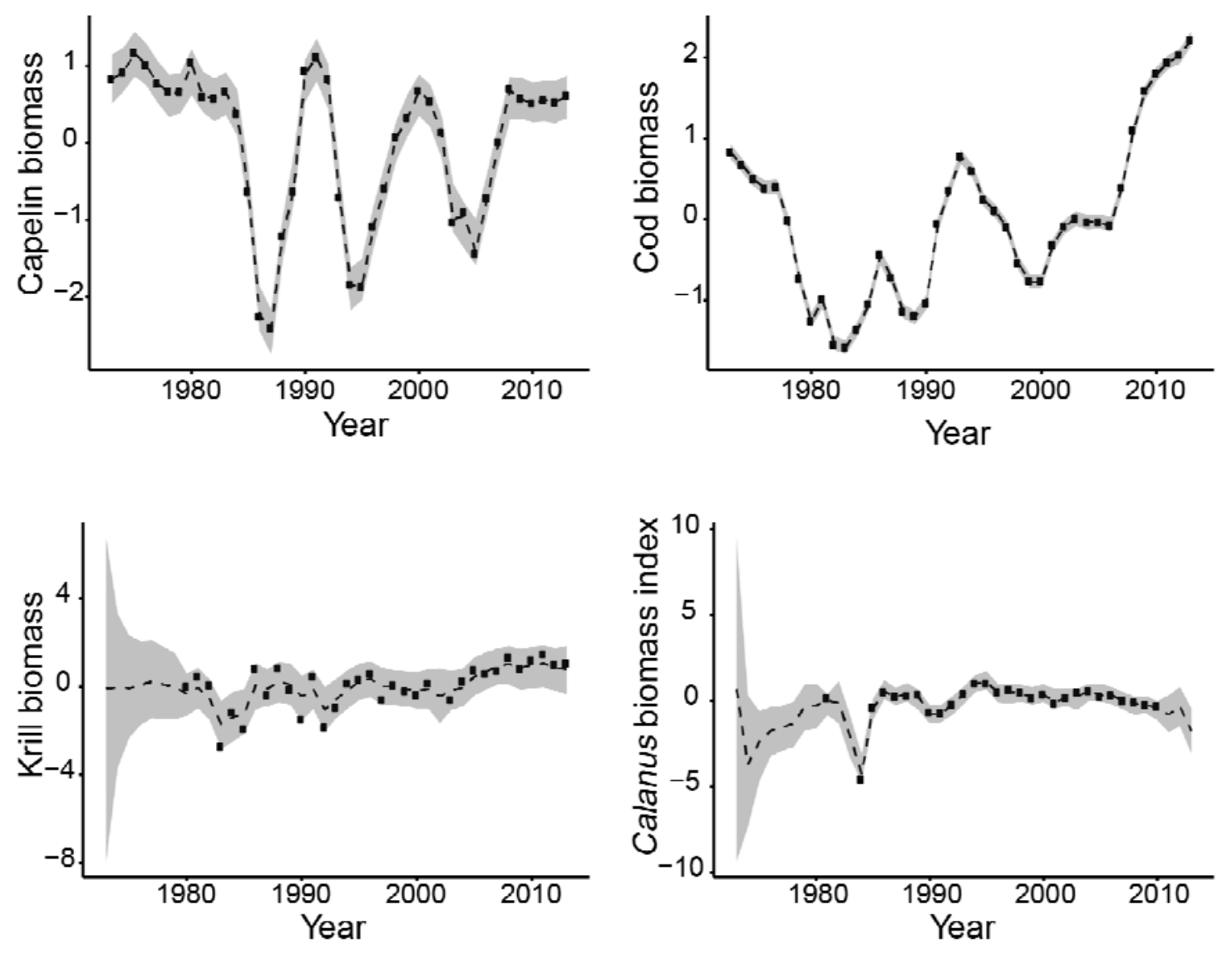

Extended Data Figure 3: Annual variation in log transformed and normalized biomass of capelin, cod, krill and, Calanus in the Barents Sea. The dots represent the observed data, the dashed lines the model posterior medians and the shaded areas the $95 \%$ credible intervals. 


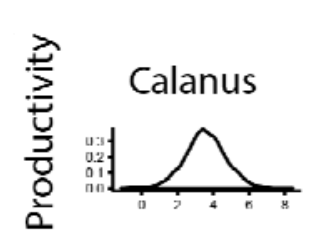

\title{
Effect on (time $t+1)$
}
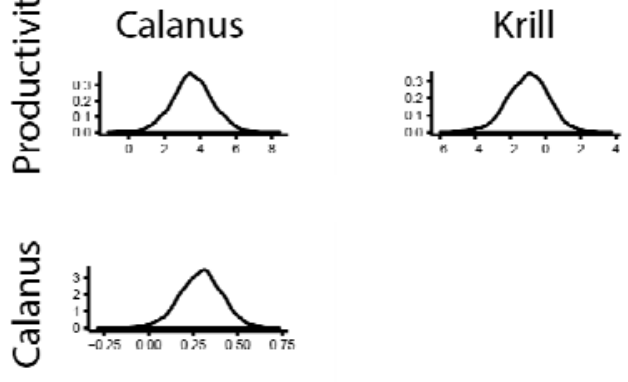

\author{
Capelin
}
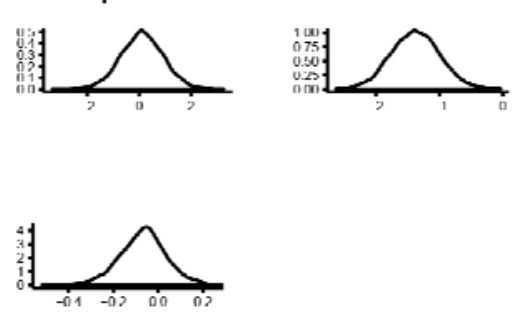

$\underset{\overline{\underline{\Sigma}}}{\overline{\underline{\Sigma}}}$
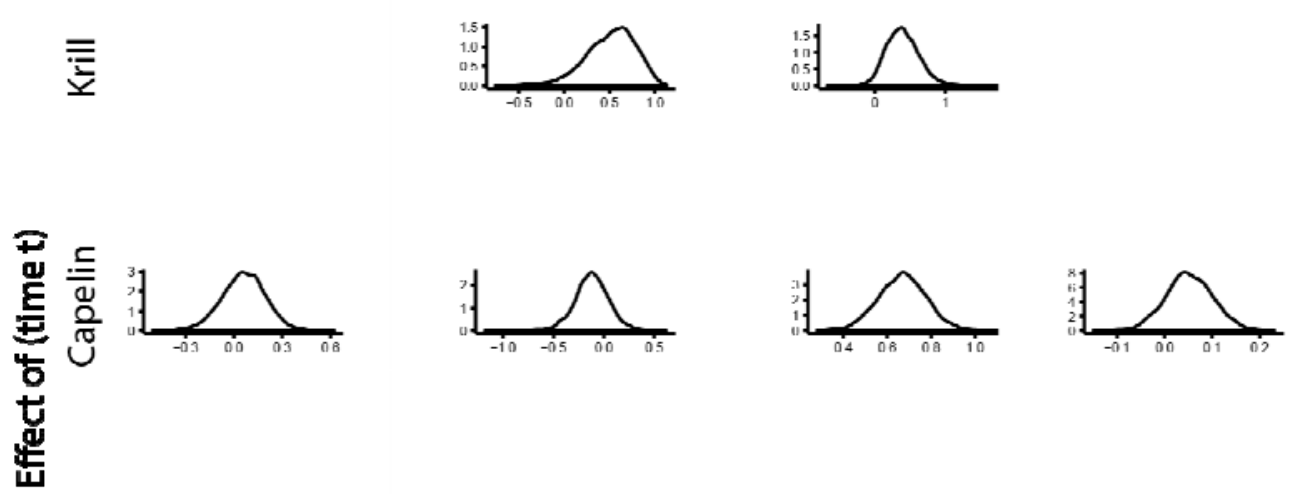

రั
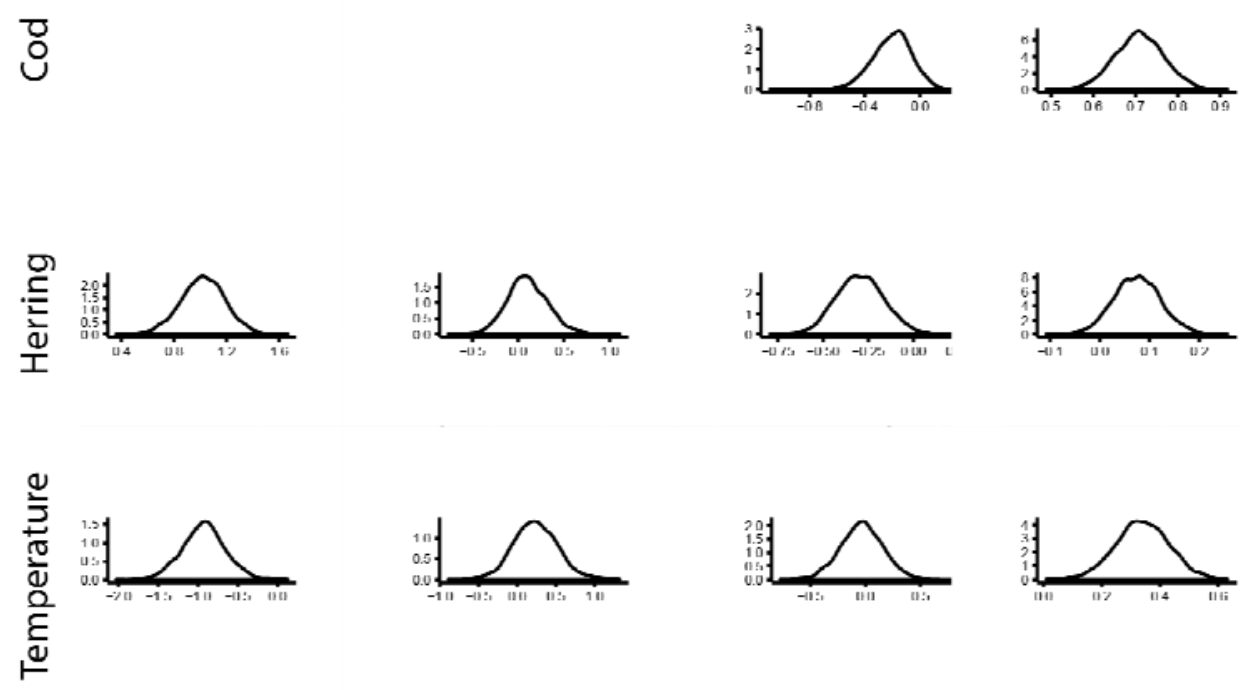

$\frac{\text { 믄 }}{\frac{\sqrt{n}}{4}}$
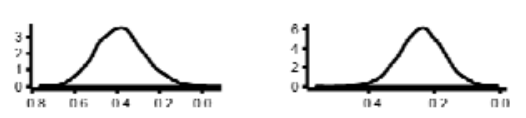
$a$

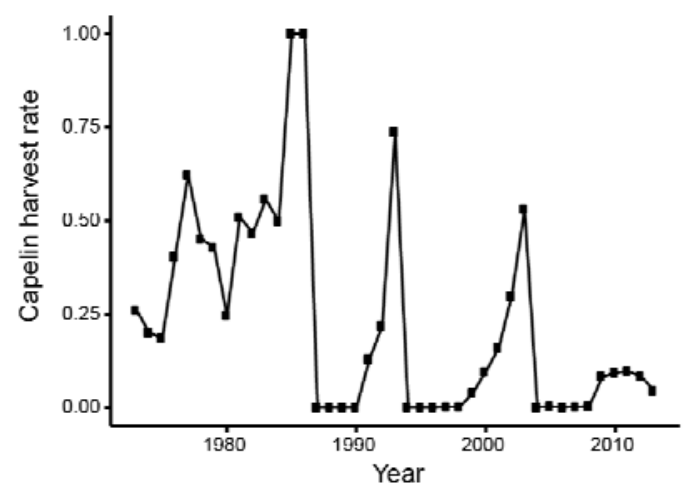

$b$

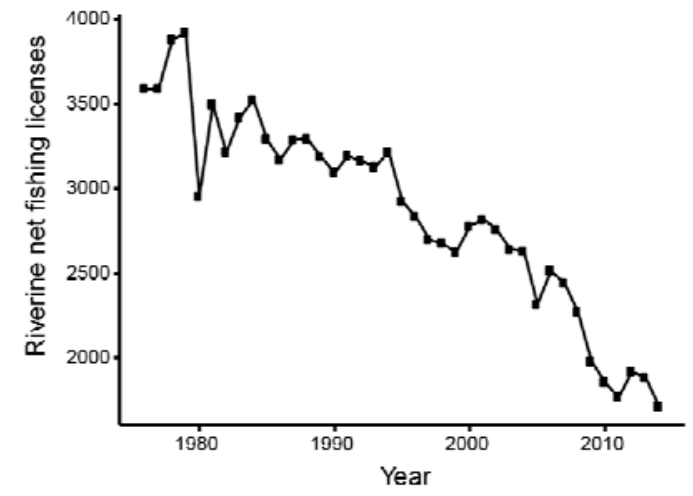

Extended Data Figure 5: Temporal variation in fishing related indices. $a$, capelin harvest rate. $b$, corrected riverine net fishing licenses (number of licences multiplied by effective number of fishing day per week).

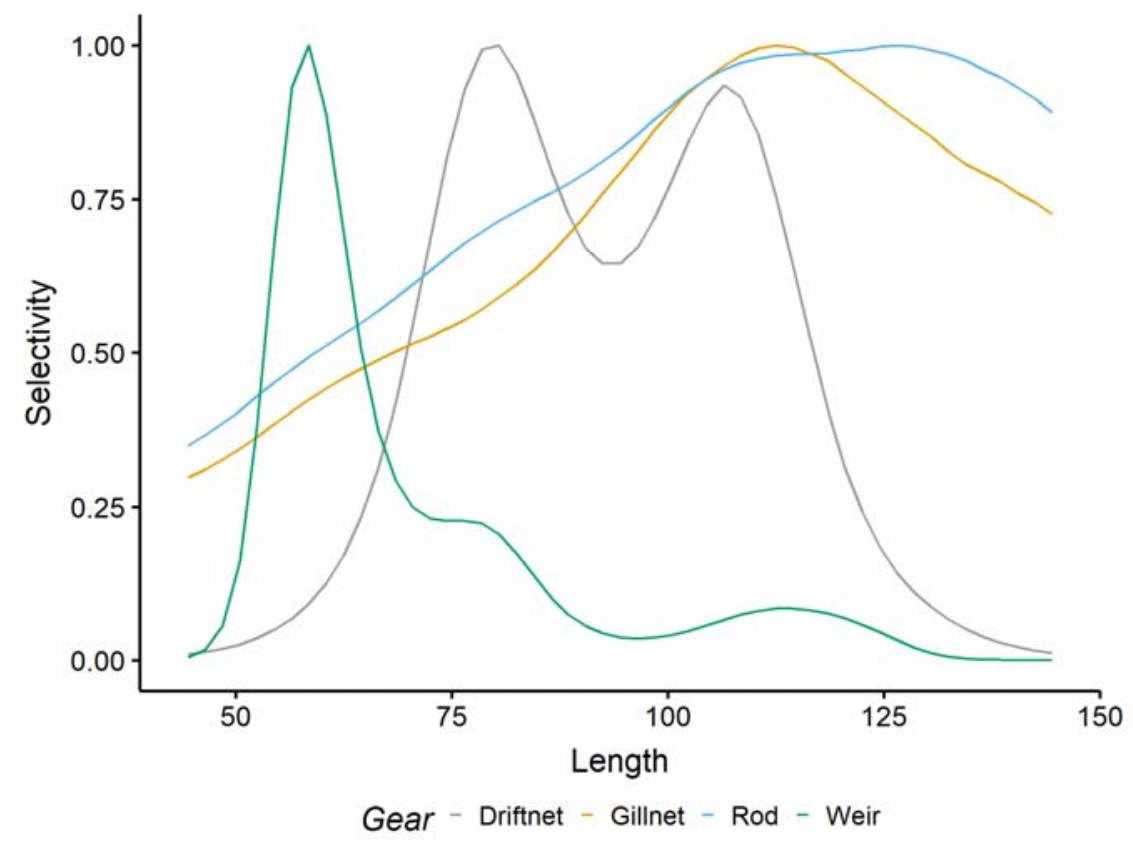

Extended Data Figure 6: Bayesian posterior median selectivity of the different gears as a function of salmon length (in $\mathbf{~ c m}$ ). Median capture probabilities were transformed so that selectivity curves peak at 1 . 

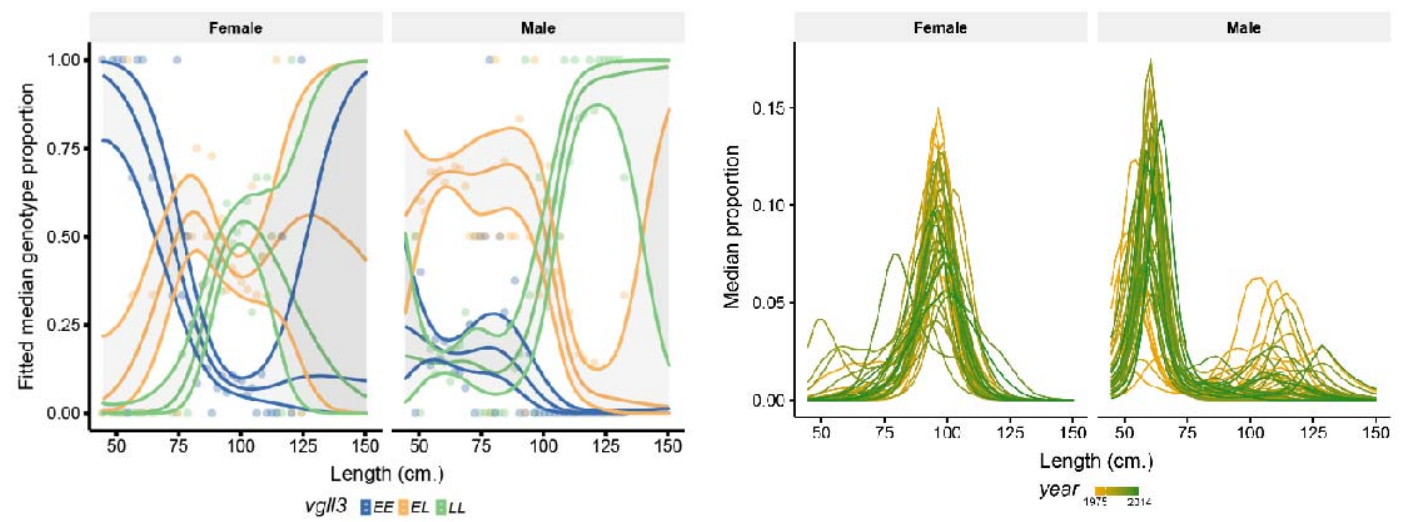

Extended Data Figure 8: Median proportion of $a$, vgll3 genotypes per length class and sex and $b$, salmon per length class, sex and year. Median proportions were calculated using metropolis Hastings sampling from $\mathbf{a}$, a multinomial GAM and $\mathbf{b}$, a negative binomial GAM (6000 iterations kept). Shaded area represents 95\% confidence intervals.

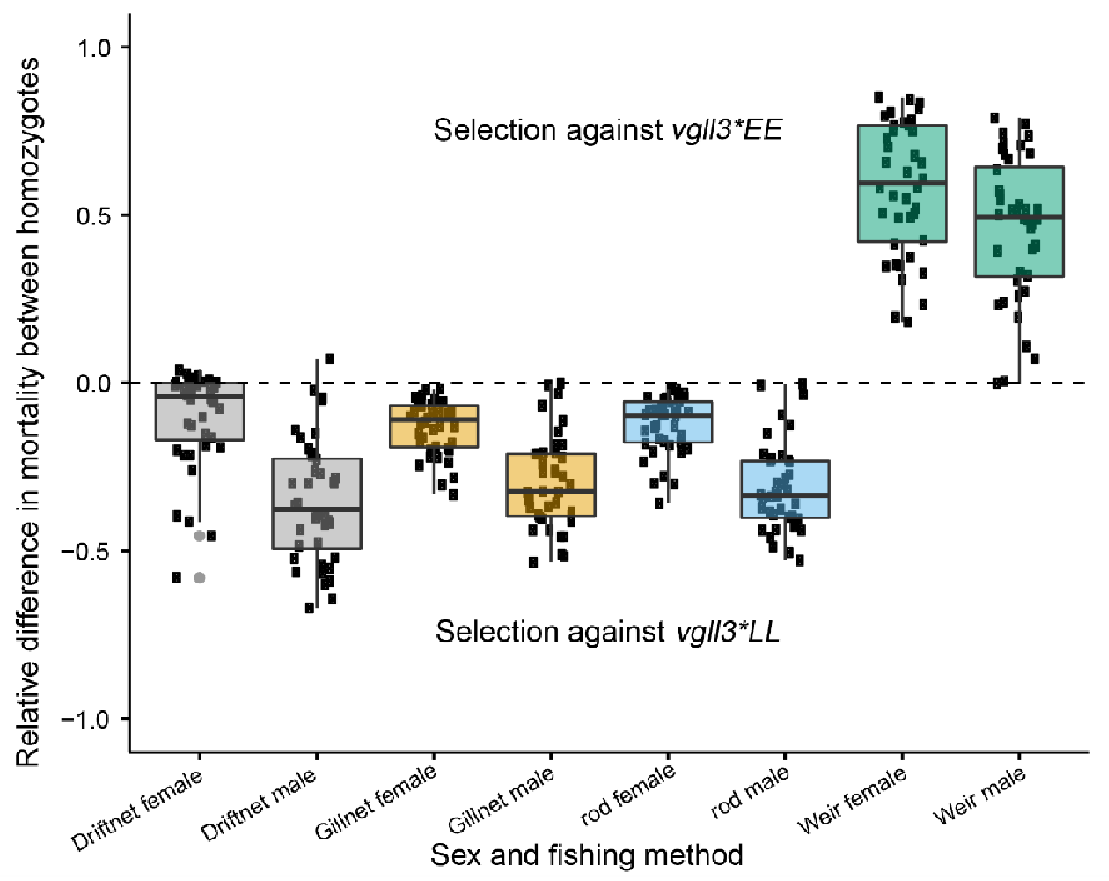

\section{Extended Data Figure 7: Median annual relative difference in mortality for} different fishing gears in males and females. Positive values indicate higher mortality in the $v g l l 3 * E E$ genotype than in the $v g l l 3 * L L$ genotype, negative values represent higher mortalities in the $v g l l 3 * L L$ genotype. Boxplots display the minimum value (median -1.5 interquartile range), $25^{\text {th }}$ percentile, median, $75^{\text {th }}$ percentile and the maximum value (median +1.5 interquartile range). 
Extended Data Table 1: AICc relative importance (RI), model averaged standardized estimates, unconditional standard errors (SE), and hypothesis testing F-tests for the 7 predictors included in the vgll3 allele frequency (quasi)binomial regressions.

Det. - de-trended.

\begin{tabular}{|c|c|c|c|c|c|c|c|}
\hline & $\mathrm{RI}$ & $\begin{array}{c}\text { Estimate } \\
\text { (log-odds) }\end{array}$ & SE & $\mathrm{F}_{(1)}$ & P-value & $\begin{array}{c}\mathrm{F}_{(1)} \\
\text { (det.) }\end{array}$ & $\begin{array}{c}\text { P-value } \\
\text { (det.) }\end{array}$ \\
\hline $\begin{array}{l}\text { River net } \\
\text { licenses }\end{array}$ & 1.00 & 0.40 & 0.09 & 27.79 & $<0.001$ & 14.15 & $<0.001$ \\
\hline Capelin log & 1.00 & 0.20 & 0.06 & 20.77 & $<0.001$ & 10.64 & 0.001 \\
\hline Krill log & 0.91 & 0.19 & 0.07 & 6.42 & 0.011 & 2.05 & 0.153 \\
\hline Herring & 0.88 & 0.19 & 0.08 & 9.30 & 0.002 & 3.23 & 0.072 \\
\hline $\begin{array}{l}\text { River rod } \\
\text { licenses }\end{array}$ & 0.69 & 0.15 & 0.08 & 1.55 & 0.213 & 0.62 & 0.431 \\
\hline Temperature & 0.56 & -0.11 & 0.07 & 2.88 & 0.090 & 2.63 & 0.105 \\
\hline Nets at sea & 0.37 & 0.08 & 0.08 & 0.91 & 0.341 & 0.12 & 0.725 \\
\hline Intercept & - & 0.37 & 0.04 & - & - & - & - \\
\hline Year & - & - & - & - & - & 18.98 & $<0.001$ \\
\hline
\end{tabular}


Extended Data Table 2: AICc relative importance (RI), model averaged standardized estimates, unconditional standard errors (SE), and hypothesis testing LRT-tests for the 9 predictors potentially influencing age at maturity probability in salmon individuals in the dataset (multinomial model).

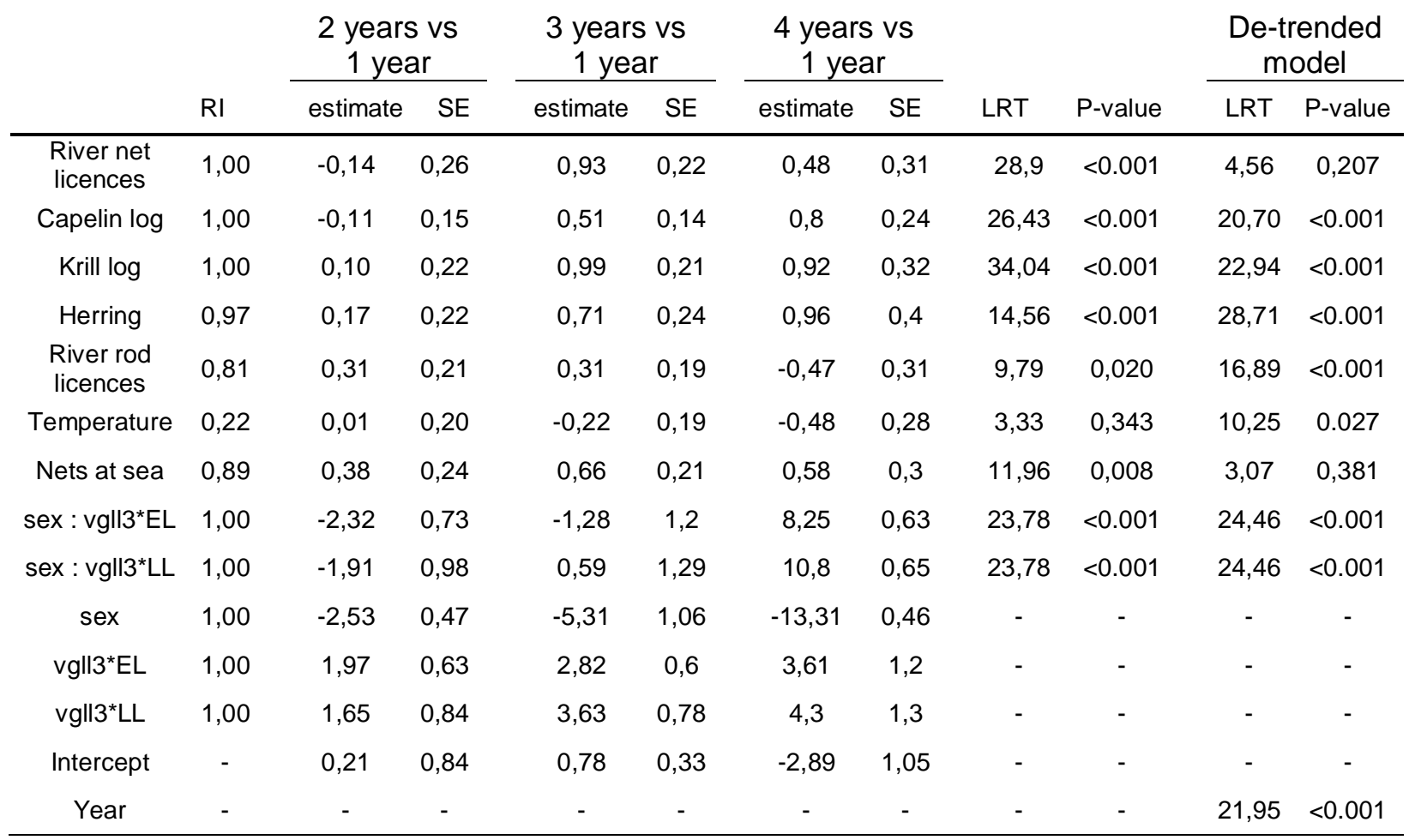

Extended Data Table 3: AICc relative importance (RI), model averaged standardized estimates, unconditional standard errors (SE) and hypothesis testing LRT-tests for the 7 predictors included in the binomial regressions for the probability to sample a female.

Det. for de-trended.

\begin{tabular}{|c|c|c|c|c|c|c|}
\hline RI & $\begin{array}{c}\text { Estimate } \\
\text { (log- } \\
\text { odds) }\end{array}$ & SE & $\operatorname{LRT}_{(1)}$ & P-value & $\begin{array}{l}\mathrm{LRT}_{(1)} \\
\text { (det.) }\end{array}$ & $\begin{array}{c}\text { P-value } \\
\text { (det.) }\end{array}$ \\
\hline
\end{tabular}

\begin{tabular}{cccccccc}
\hline River net licences & 1.00 & 0.52 & 0.12 & 22.32 & $<0.001$ & 0.35 & 0.553 \\
Capelin log & 1.00 & 0.40 & 0.07 & 34.06 & $<0.001$ & 14.28 & $<0.001$ \\
Krill log & 1.00 & 0.44 & 0.11 & 15.94 & $<0.001$ & 10.79 & 0.001 \\
Herring & 1.00 & 0.50 & 0.11 & 20.84 & $<0.001$ & 24.86 & $<0.001$ \\
River rod & 0.30 & 0.07 & 0.11 & 0.55 & 0.458 & 4.53 & 0.033 \\
licences & 0.32 & -0.07 & 0.10 & 0.42 & 0.517 & 1.70 & 0.192 \\
Temperature & 1.00 & 0.51 & 0.11 & 25.20 & $<0.001$ & 16.78 & $<0.001$ \\
Nets at sea & - & -0.48 & 0.06 & - & - & - & - \\
Intercept & - & - & - & - & - & 37.27 & $<0.001$ \\
Year & - & - & & & & & \\
\hline
\end{tabular}




\section{Supplementary notes}

596 Drivers of $\boldsymbol{v g l l 3}$ allele frequency changes

597 The maximum variance inflation factor calculated, for the number of nets at sea, was 3.79 , below

598 the frequently recommended thresholds. The top ranked model based on the AICc had an Akaike

599 weight of 0.22 , indicating model uncertainty. It included the number of riverine net and rod

600 licenses, capelin, krill, herring (log) biomasses and temperature covariates. The number of riverine

601 net licenses, capelin, krill and herring were all included in the 5 other models within a $\triangle \mathrm{AICc}$

602 inferior to two. Model parameters were averaged with the conditional method over 16 models

603 having a summed weight of 0.95 (Extended data Table 1). The dispersion parameter was 0.89 in the

604 full model.

\section{Genetic and plastic basis of phenotypic changes}

606 Using a multinomial regression, we investigated the role of the environmental variables on the 607 probability to observe the different age at maturity classes. After controlling for the sex-specific 608 genetic effects, we found that the variables positively associated with $v g l l 3 * L$ were also positively 609 associated with the probability to observe older, later maturing Atlantic salmon (Extended Data 610 Table 2). The total number of nets at sea was also positively associated with later maturation 611 probabilities $\left(\chi_{(3)}^{2}=11.96\right.$, P-value $=0.008$, Extended Data Table 3$)$. However, the number of nets 612 at sea and the number of riverine net fishing licenses were no longer significant in the de-trended 613 regression $\left(\chi_{(3)}^{2}=3.07\right.$, P-value $=0.381, \chi_{(1)}^{2}=4.56$, P-value $=0.207$, respectively; Extended Data 614 Table 2). The model obtained with backward variable selection also obtained the highest AICc, with 615 a $\triangle$ AICc superior to 3, indicating strong support. Model averaging was performed over 7 models 616 (Extended Data Table 2).

\section{Proportion of females}

618 Using a binomial model, we found that variables positively associated with the $v g l l 3 * L$ allele 619 frequency were also positively associated to the probability to sample a female (Supplementary 620 notes; Extended Data Table 3, Extended Data Figure 2). The number of nets at sea was also 621 positively correlated with female proportion $\left(\widehat{\beta}^{*}=0.50, \chi_{(1)}^{2}=25.20\right.$, P-value $<0.001$, Extended 622 Data Table 3). Temporal variation in number of nets at sea is particularly influenced by the 623 cessation of marine driftnet fishing in 1989, the fishing method the most used in the previous period 624 and selecting preferentially $1 \mathrm{SW}$ and small $2 \mathrm{SW}^{42}$, corresponding mainly to males in Tenojoki. In 625 the de-trended model, the number of riverine net licenses was not significantly associated with 626 female proportion anymore (Extended Data Table 3), however the number of rod fishing licenses 627 became marginally positively associated with the probability to sample a female (Extended Data 628 Table 3). Four models cumulating an AICc weight greater than 0.95 were used in the model 629 averaging.

\section{Multispecies Gompertz model}

631 The multispecies Gompertz model reproduces the observed variation in biomass indices well 632 (Extended Data Fig. 3). The correlation coefficients between the observed and modelled biomass 633 indices were greater than 0.91. The negative effect of fishing on capelin biomass (Extended Data 634 Fig. 4) was used to quantify the indirect effect on $v g l l 3$ allele frequency. 


\section{Net fishing selection}

\section{Gear selectivity}

637 Selectivity estimated from sonar data indicated specific patterns for each gear type (Extended Data 638 Fig. 6). Weir selectivity was bell-shaped with a modal value of $58 \mathrm{~cm}$. Driftnet selectivity was 639 bimodal with modal values of 80 and $107 \mathrm{~cm}$. Gillnet and rod had sigmoid selectivity curves with 640 capture probabilities increasing with salmon length until reaching maxima at 112 and $126 \mathrm{~cm}$, 641 respectively.

\section{Probabilities of vgll3 genotypes per length class}

643 The probability of vgll3 genotypes in harvested salmon differed according to the $2 \mathrm{~cm}$ length 644 classes and sexes (Extended Data Fig. 8a). Small females (e.g. below $75 \mathrm{~cm}$ ) were more likely to be $645 v g l l 3^{*} E E$ than $v g l l 3^{*} E L$ and $v g l l 3 * L L$, while small males were more likely to be $v g l l 3 * E L$ and had a 646 similar probability to be $v g l l 3 * L L$ and $v g l l 3 * E E$.

\section{Length-frequency distributions}

648 The median length-frequency distributions of harvested salmon calculated from negative binomial 649 GAM fitted values differed between sexes (Extended Data Fig. 8b). Females measuring around 97 $650 \mathrm{~cm}$ were the most frequent whereas most frequent males measured around $60 \mathrm{~cm}$. There was 651 among-year variation in the length-frequency distribution, particularly in males, where large 652 individuals were more frequent in the early years of the time series. The negative binomial 653 parameter controlling the over-dispersion was 7.31.

\section{Relative fishing mortality per sex and gear}

655 The expected relative difference in fishing mortality between alternative vgll3 homozygotes varied 656 greatly among gears, sex and years (Extended Data Fig. 7). On average, the annual relative 657 difference in mortality was higher in males than females for fishing gears selecting against vgll $3 * L L$ 658 (i.e. driftnet, gillnet and rod) but lower for weir fishing selecting against $v g l l 3 * E E$. Fishing 659 mortality of $v g l l 3 * L L$ was on average $52 \%$ lower than $v g l l 3 * E E$ when weir was used but $27-29 \%$ 660 higher when driftnet, gillnet or rod were used. The harvest rate had a negligible effect on the 661 calculated difference in mortality between homozygotes. The annual medians calculated with a 662 harvest rate of 0.5 differed, on average, by $1 \%$ from those calculated with harvest rates of 0.4 or 0.6 .

\section{Proportion of females per year and their vgll3 genotypes}

664 The GAM smooth term estimating temporal variation in female proportion was not significant for 665 the $E E$ genotype (edf $=0.45, \mathrm{~F}=0.05, \mathrm{P}$-value $=0.171$ ) where the female proportion declined 666 linearly over time. The proportion of $L L$ females decreased over time in a slightly non-linear 667 manner $(\mathrm{edf}=1.32, \mathrm{~F}=0.33$, P-value $=0.001$ ) whereas the proportion of $E L$ females mainly 668 declined during the 1980s and the last 7 years of the time series (edf $=6.25, \mathrm{~F}=2.07$, P-value < 669 0.001). The highest change in female proportion was observed for $E L$ heterozygotes which was also 670 the genotype with the largest difference in age at maturity between sexes, due to sex-specific 671 dominance patterns. The dispersion parameter of the quasi-binomial GAM was 1.52. 


\section{Temporal variation in relative mortality and selection}

673 The total harvest rate in the Teno river, fixed at plausible values $(0.4,0.5$ or 0.6$)$, would be strong 674 enough to induce significant net fishing induced selection (i.e. differences in survival between $675 v g l l 3^{*} E E$ and $v g l l 3^{*} L L$ individuals; Fig. 3). The annual variation in net selection was due to 676 changes in the sex-specific length-frequency distributions, the relative use of the different fishing 677 gears and the sex ratio. Regardless of the harvest rate, the median selection values were all positive 678 and indicated net fishing selection against vgll3*EE individuals. Confidence intervals didn't overlap 679 zero in 20 of the 40 years, independently of harvest rates. The median selection coefficients were on 680 average 2.37-2.70 times higher in the first 10 years of the time series than in the last 30 years.

681 The median estimates of selection coefficients calculated under a harvest rate of 0.5 were included 682 into the vgll3 quasi-binomial model as an independent variable. The calculated fishing selection was 683 positively associated with the vgll3*L allele frequency $\left(\widehat{\beta^{*}}=0.13, \mathrm{CI}_{95 \%}=[0.03,0.22], \mathrm{F}_{(1)}=4.87\right.$, 684 P-value $=0.027)$. The significance of other variables remained unchanged. After backward 685 selection, the model included net fishing selection, riverine net fishing licenses $\left(\widehat{\beta^{*}}=0.36, \mathrm{~F}_{(1)}=\right.$ 686 18.52, P-value $<0.001)$, capelin $\left(\widehat{\beta^{*}}=0.20, \mathrm{~F}_{(1)}=14.48\right.$, P-value $\left.<0.001\right)$, herring $\left(\widehat{\beta^{*}}=0.21, \mathrm{~F}_{(1)}=\right.$ 687 11.50, P-value $<0.001)$ and krill $\left(\widehat{\beta^{*}}=0.15, \mathrm{~F}_{(1)}=4.54, \mathrm{P}\right.$-value $\left.=0.033\right) \log$-biomass. The 688 dispersion parameter was 0.88 .

689 The quadratic trend in the net fishing selection was removed to refit the vgll3 quasi-binomial model

690 on the residuals (year $\widehat{\beta^{*}}=-0.03, \mathrm{CI}_{95 \%}=[-0.05,-0.02]$, year ${ }^{2} \widehat{\beta^{*}}=0.02, \mathrm{CI}_{95 \%}=[0,0.04]$ ). Riverine 691 net fishing licenses, the net fishing selection and capelin log-biomass remained significant in the de692 trended model $\left(\widehat{\beta^{*}}=0.18, \mathrm{~F}_{(1)}=15.60\right.$ and $\mathrm{P}$-value $<0.001, \widehat{\beta^{*}}=0.14, \mathrm{~F}_{(1)}=8.97$ and $\mathrm{P}$-value $=$ $6930.003, \widehat{\beta^{*}}=0.12, \mathrm{~F}_{(1)}=7.47$ and P-value $=0.006$; respectively $)$ including a year effect $\left(\widehat{\beta^{*}}=-0.18\right.$, $694 \quad \mathrm{~F}_{(1)}=15.17$ and $\mathrm{P}$-value $\left.<0.001\right)$. 